\title{
The virtual small cells based on UE positioning: a network densification solution
}

\author{
Thomas Varela Santana* (D), Sofía Martínez López and Ana Galindo-Serrano
}

\begin{abstract}
Next wireless generation mobile networks will be composed of a large number of antennas at the base station (BS), which is known as massive multiple input multiple output (MIMO). Thanks to this technology, the BS can focus the energy on a user equipment (UE) or group of UEs to improve their throughput and the network capacity. We call these coverage areas virtual small cells (VSCs). Their main advantage is that they allow increasing the network capacity through a virtual densification, therefore, avoiding the deployment cost of new infrastructure. Identifying the dense traffic areas in real time and providing a good quality of service arises as a key challenge to be addressed. Our work focuses on the interaction between the VSCs and the identification of the dense traffic areas, where a feedback scheme is proposed. This feedback scheme is based on the location of these dense traffic areas provided by our proposed clustering methods. To conduct this research, we propose (i) a VSC architecture and system model with a specific codebook in order to avoid feedback overhead, and (ii) two positioning algorithms in order to determine the hotspots localization. The first positioning algorithm is based on K-means method and is centralized at the BS using Global Positioning System (GPS) coordinates, and the second one is based on cooperative communications using ultra-wideband (UWB) signals in order to avoid the network participation. Finally, simulations of these positioning methods intended for the use of the VSCs are presented. These results show significant improvement compared to already existing methods. Furthermore, these positioning methods highly reduce the feedback since, accordingly to our VSC model, the BS only requires angles information based on the localized hotspots.
\end{abstract}

Keywords: Massive MIMO, VSC, GPS, UWB, D2D, Positioning, Cluster localization

\section{Introduction}

Ensuring constantly growing and changing capacity requirements with low infrastructure investment in mobile network is a key challenge for operators. The fourth-generation (4G) considers the introduction of small cells in addition to the base stations (BSs) to deal with dense traffic, as small cells help focusing energy on a given area such as commercial centers, stadiums, etc. Nevertheless, the use of small cells represents high investment for operators as they involves important capital expenditure (CAPEX), i.e. backhaul deployment, site acquisition and also operational expenditure (OPEX), as energy consumption and maintenance $[1,2]$. This is justified when capacity requirements are stable over time and space.

*Correspondence: thomas.varelasantana@orange.com Orange Labs, 44 Avenue de la République, 92320 Châtillon, France
Different challenges have been identified in future wireless communications. An important envisaged requirement is to provide great service in a crowded network [3] in a more energy-efficient way. Some examples of possible scenarios are meeting rooms at certain hours in business buildings, shopping, stadiums, open air festivals, public events, traffic jams, etc. Notice that the occurrence of these scenarios is non-predictable by the operator. The optimal solution, from the infrastructure point of view, could be to adapt the coverage of these unpredictable crowded areas, in terms of time and localization, as they are at the origin of dense traffic. The idea is to have an elastic network infrastructure. An example of this, is the Google Loon project [4], where balloons equipped with antenna systems placed at the stratosphere at around $20 \mathrm{~km}$ from the surface of the earth and steered using the different wind current layers are used to provide coverage. 
In order to avoid the small cells, two-dimensional (2D) large antenna array at the BS named massive multiple input multiple output (MIMO) can be exploited.

Adding more antennas at the BS provides more degree of freedom to the propagation channel between the transmitter and the receiver. Due to these degrees of freedom, higher diversity, and higher data rate can be achieved, increasing also the capacity [5-7]. Improvement of energy efficiency in cellular system using an increased number of antennas at the transmitter has been studied and explained in [6] (and references therein). With the use of 2D antenna elements highly directive beams to cover a given area can be created [8]. We call this concept virtual small cell (VSC) which consists of highly directive beam working in co-channel that point to cluster estimated hotspot, increasing the signal interference noise ratio (SINR) with massive MIMO at the BS and without small cells [9]. Reduction of co-channel interference by using an unlicensed band for the VSCs is proposed in [10]. Security and caching aspects for VSCs have been considered in $[11,12]$ respectively, while ergodic sum-rate has been derived in [13] for VSCs.

The benefits of VSCs compared to three-dimensional (3D) user equipment (UE)-specific beamforming (for massive MIMO) are the following:

- VSC allows to dynamically allocate the radio resources for each geographical zone according to the dynamic spatial traffic.

- VSCs are transparent for UEs, and can then be used for legacy UEs. On the contrary, UE-specific beamforming requires UEs that support the number of extend codebooks.

- VSCs are more robust to UE mobility and/or channel variations since the focalization area is wider. If the 3D UE-specific beamforming is narrow, the motion of the UE forces to rapidly update the precoding in order to refocus the energy on the new UE location.

A way to implement the VSCs according to the UEs position is to firstly evaluate the cellular traffic. Secondly, based on the previous cellular traffic analysis, the hotspots of UEs have to be identified. If the detected hotspot is new in the cell, and it respects the constraint location, a highly directive beam will be focused on it, i.e., a VSC is implemented. If hotspots in the database have disappeared, the associated VSCs are removed. By repeating these previous steps, we obtain a periodical methodology which gives us a general view on when to implement or eliminate the VSCs. This methodology has been presented in one of our previous works [14] and is summarized in Fig. 1 for reading clarity. Concerning the constraint locations, the performance of the VSC can vary depending on its steering point. There are basically two constraints to consider, the VSC steering point should not be too close to the macrocell and should not be at the bore-sight. This last point is more detailed and represented in Fig. 3 in Section 3.

To implement a VSC, the directive beam is guided thanks to a well design precoder. Precoding is a generalization of beamforming to support multi-stream transmission in multi-antenna wireless communications.

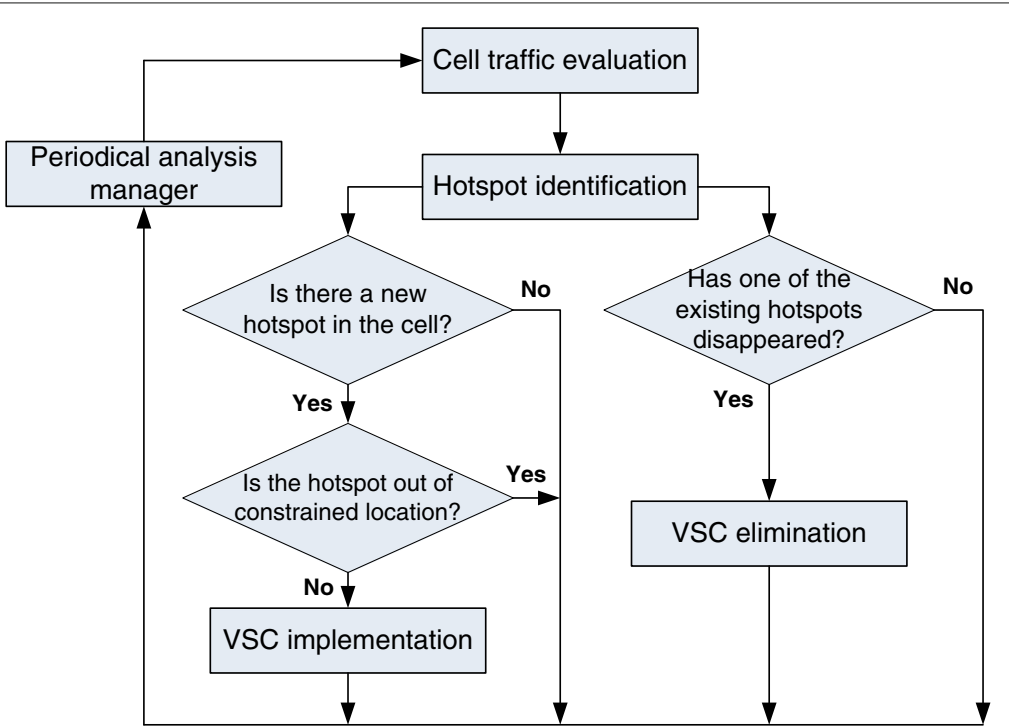

Fig. 1 VSC configuration methodology. This figure represents a periodical methodology which allows the implementation and elimination of the VSC. It consists of a cell traffic evaluation, then in a second phase, we identify the hotspots of UEs based on the previous cellular traffic analysis. If the detected hotspot is new in the cell, a highly directive beam will be focused on it, i.e., a VSC is implemented. If hotspots in the database have disappear, the associated VSCs are removed 
Actually, such a precoder will be chosen depending on a predefined codebook based on specific characteristics of the detected hotspots. These characteristics are the center and the radius of the corresponding hotspot. Depending on operator strategies, several clustering methods have been developed for positioning in fifth-generation (5G) [15] in order to identify such a hotspot. One strategy is to centralize all the information at the BS [14] and another is to identify the hotspots without intervention of the BS by using direct device-to-device (D2D) link between all the UEs [16]. Both methods need synchronization whether at the BS or between the UEs [17]. Once a hotspot has been identified, an easy feedback can be performed in order to select the adequate precoder for the beam formation. Smooth feedback information is essential as the large number of antennas made the channel state information (CSI) very important and may cause huge amount of feedback overhead as each antenna needs a feedback from each device. In time division duplexing (TDD) mode, the reciprocity of the downlink and uplink could be used for channel estimation but reciprocity is not available in frequency division duplexing (FDD) mode where uplink and downlink occurs in separated bands. VSC requires less radio overhead for FDD. Indeed, the number of antenna ports for the case of VSC is reduced compared to the case of massive MIMO. In massive MIMO, one different downlink reference signal is needed for each antenna port $[18,19]$. Therefore, the more antenna ports, the more radio signal overhead is needed at transmission. The feedback of VSC is smaller than the one required for massive MIMO while still able to track changes in the traffic generated by the group of served UEs. Our proposed feedback is based on index grouping transmission, where one index is selected from the codebook for each detected hotspot. Once the index information is available at the BS, we can select the adequate precoder and create the VSC.

UE positioning applied to $5 \mathrm{G}$ cellular management networks has been deeply described for different scenarios in [20] and also an architecture improving time response using location for indoor scenarios is presented in [21]. VSCs could be also deployed in indoor scenarios, where nowadays small cells are more commonly used. The home/office scenario is given by the constant and recurrent human behavior, i.e., switching from houses to offices during the day, to shopping malls and restaurant areas. In this case, the traffic flow is predictive and therefore, operators can know in advance the occurrence of a high traffic demand in a given period of the day at a given location. To fulfill the capacity demand in the crowded areas, operators can previously program the activation and deactivation of VSCs pointing to the identified areas in the adequate periods. Also, giving coverage to deep indoor scenarios could be possible thanks to the high gain achieved by VSCs. This would substitute the installation of fix femtocells. Also, operators could cover specific floors in business buildings with VIP users having a punctual meeting or hosting an event.

The contributions of this work are the following:

- Whereas VSC is not a new topic, this paper is the first to provide explicit mathematical description of VSC implementation. In particular, we extend and apply the "one-ring" model to the VSCs and express the corresponding correlation matrix.

- The paper highlights the importance of hotspot location knowledge to efficiently implement VSCs. Indeed, the correlation matrix, number of antennas, codebook, and feedback of the VSC are strongly dependent on the hotspot location.

- Due to this hotspot location dependence, the paper proposes to study and compare hotspots location techniques by firstly generalizing a GPS-based method from [14] and secondly a cooperative UWB method.

- Finally, the paper gives a methodology on how to implement VSC in the real network by comparing different approaches for the steering of VSC.

The rest of this paper is organized as follows: in Section 3, we start defining the system model and the architecture of the VSC with SINR evaluation. Section 4 extends our model to the case of multi-VSCs by generalizing our system model and by proposing a codebook design for feedback scheme. Two methods for group localization are detailed in Section 5, one based on BS centralized strategy, and another distributed approach based on direct communication between the devices independently of the BS. We present the results in Section 6 and end by a conclusion in Section 7.

\section{Method section}

In this article, we first design the angles between the BS and the hotspot of UEs to identify. These angles are characterizing the beam. Then, the number of antennas has been also defined depending on the angles above. The system model for radio communication has been mathematically derived, first considering only one VSC and secondly considering multigroup of UEs, where the received signal and the SINR are expressed. A feedback based on codebook design is then proposed, which highly reduce the feedback overhead. The codebook only depends on the characteristics of the hotspots, which are the radius and the center localization. These characteristics are provided by two possible localization methods that are explained in the article. The first one is based on GPS positioning and use $\mathrm{K}$-means algorithm. The second one is based on UWB positioning and use ToA estimation. Both methods 
have been compared in an outdoor LOS environment, and the UWB-based method provides better accuracy results, error estimation smaller than $1 \mathrm{~cm}$ in $50 \%$ of the cases.

\section{Virtual small cell architecture and system model}

We consider a specific area where major part of the UEs are localized inside. VSC could be implemented by common BSs, as long as the number of antennas is enough to steer the beam as required [9]. Note that there are two options when using a VSC. The first one is associating a different cell ID to the VSC with respect to the cell. This would correspond to network densification without new equipment deployment. The second one is to use the same cell ID (also same frequencies and mobility management) as that of the cell. In this case, the term VSC could be replaced by beam but would work similarly. This depends on the actual implementation of VSC, and particularly whether a different cell ID is given or not.

Figure 2 describes the angles in the horizontal and vertical planes that are used for building a VSC. The VSC is hence defined by its steering $\theta_{\text {tilt }}$ and his beamwidth $\theta_{3 \mathrm{~dB}}$ in the vertical plane as presented in Fig. 2a. Those two angles can be respectively implemented using simple trigonometric functions:

$$
\begin{aligned}
& \theta_{\mathrm{tilt}}=-\arctan \left(\frac{h}{\text { dist }}\right)[\mathrm{rad}] \\
& \theta_{3 \mathrm{~dB}}=\arctan \left(\frac{h}{\operatorname{dist}-r}\right)-\arctan \left(\frac{h}{\mathrm{dist}+r}\right)[\mathrm{rad}]
\end{aligned}
$$
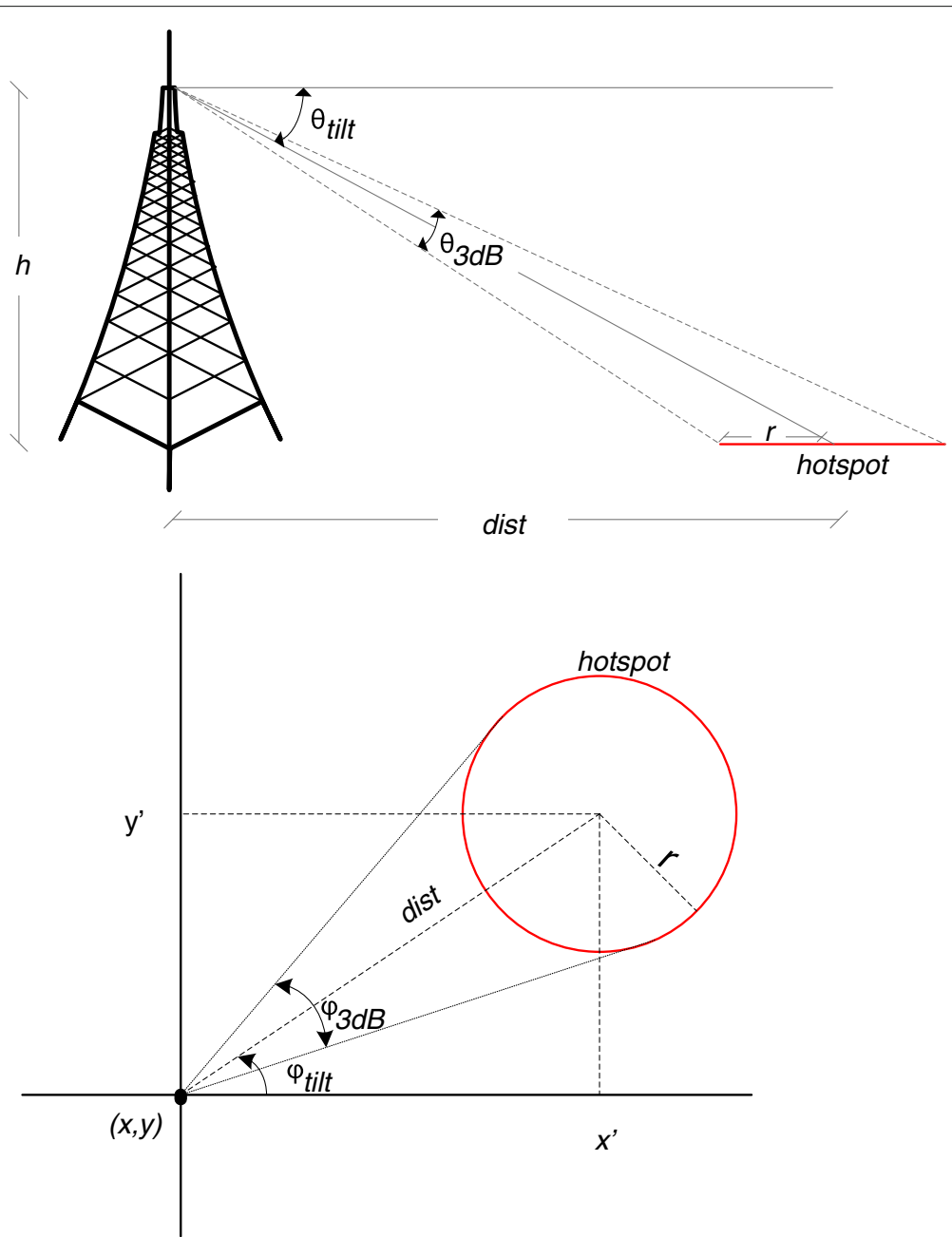

Fig. 2 Beamwidth and beam steering in the vertical and horizontal planes. This figure represents the architecture of a BS with the angles in the horizontal and vertical planes that are used for building this VSC. Figure 2a represents the angles of the beam in the vertical plane. And Fig. $2 \mathrm{~b}$ represents the angles of the beam in the horizontal plane 
where dist is the projection in the vertical plane of the distance between the BS and the center of the localized hotspot, $h$ is the height of the BS and $r$ is the hotspot radius. From the horizontal plane, we can distinguish two other angles, $\varphi_{\text {tilt }}$ and $\varphi_{3 \mathrm{~dB}}$ as represented in Fig. $2 \mathrm{~b}$ and expressed using trigonometric functions as follows:

$$
\begin{aligned}
\varphi_{\mathrm{tilt}} & =-\arctan \left(\frac{y-y^{\prime}}{x-x^{\prime}}\right) \quad[\mathrm{rad}] \\
\varphi_{3 \mathrm{~dB}} & =2 \arctan \left(\frac{r}{\mathrm{dist}}\right)[\mathrm{rad}]
\end{aligned}
$$

where $\left(x^{\prime}, y^{\prime}\right)$ are the coordinates of the located hotspot and $(x, y)$ the coordinates of the BS.

The antenna elements (i.e., $N_{v}$ and $N_{h}$ antennas in the vertical and horizontal plane, respectively), used for creating a beam, depends on the angles above. They also depend on the array element distance $\lambda D$, where $\lambda$ is the wavelength factor and on the value 50 that has been given by an antenna expert of our group based on experiments for our topology:

$$
\begin{aligned}
& N_{v}=\frac{50}{\theta_{3 \mathrm{~dB}}^{o} \lambda D \cos \left(\theta_{\text {tilt }}\right)}, \\
& N_{h}=\frac{50}{\varphi_{3 \mathrm{~dB}}^{o} \lambda D \cos \left(\varphi_{\mathrm{tilt}}\right)},
\end{aligned}
$$

The number of antenna elements at the BS is computed as:

$$
M=N_{\mathrm{mc}}+N_{\mathrm{VSC}}
$$

where $N_{\mathrm{mc}}$ are the antenna elements used for the macrocell and $N_{\mathrm{VSC}}=N_{v} \times N_{h}$ are the active antenna elements used to create a beam for the VSCs. This will automatically reduce the CSI feedback overhead because the number of antenna ports for the case of VSC is reduced compared to the case of massive MIMO $\left(N_{\mathrm{VSC}}<M\right)$.

As stated in the introduction, the performance of the VSC can vary depending on its steering point:

- If the VSC steering point is close to the macrocell, the UEs will experience high interference from the macrocell. $\theta_{\text {tilt }}$ will increase and its projection distance in the horizontal plane $d_{\theta_{\text {tilt }}}$ also, involving increased number of antenna elements in the vertical axis, as expressed in (5).

- If the VSC is steering towards the edge of the cell, i.e., if the azimuth angle $\varphi_{\text {tilt }}$ is close from bore-sight, the number of antennas required is higher as shown in (6).

These two location constraints are represented in Fig. 3, where dashed areas represent the forbidden regions where VSCs will not be deployed.

Let us consider a BS, where $M$ antennas elements are used by the VSC serving $K$ UEs working in FDD over

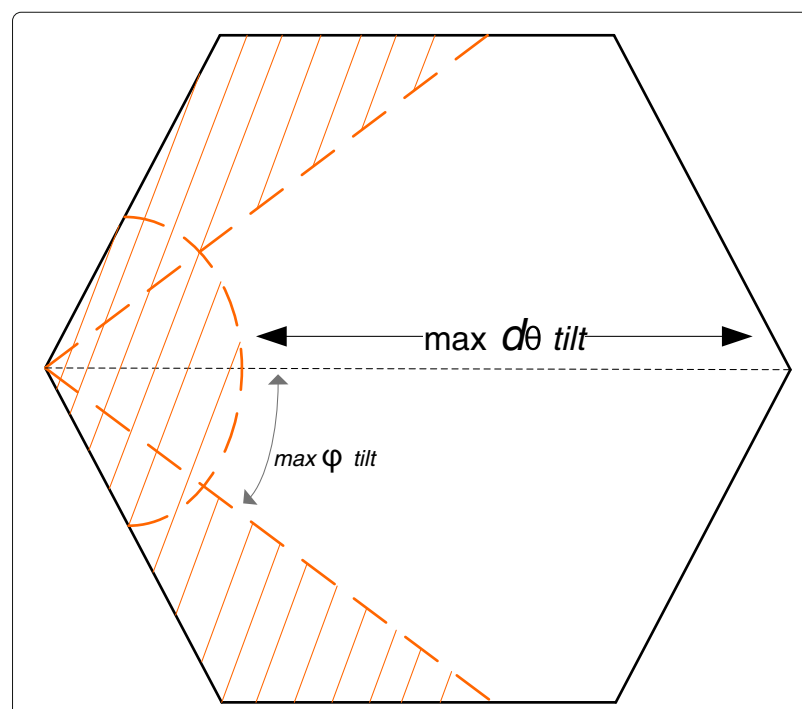

Fig. 3 Constraint location for VSC in hexagonal cell. This figure represents the constraint location in an hexagonal cell where the VSCs can not be deployed

a Rayleigh-fading channel. For each channel $\mathbf{h}_{k} \in \mathbb{C}^{M}$, $1 \leq k \leq K$, a linear precoding is employed at the BS to support simultaneous downlink transmissions to $K$ UEs. We call $\mathbf{x} \in \mathbb{C}^{M}$ the linearly precoded signal vector subject to the transmit power constraint $\mathbb{E}\left[\|\mathbf{x}\|^{2}\right] \leq P$. The received signals at the $K$ UEs is represented by the vector $\mathbf{y} \in \mathbb{C}^{K}$ and can be expressed as

$$
\mathbf{y}=\mathbf{H}^{H} \mathbf{x}+\mathbf{n},
$$

where $\mathbf{H}=\left[\mathbf{h}_{1}, \ldots, \mathbf{h}_{K}\right]$ is the downlink channel matrix of the VSC and $\mathbf{n} \sim \mathcal{N}_{\mathcal{C}}\left(\mathbf{0}, \mathbf{I}_{K}\right)$ is the Gaussian noise. Also, the transmitted signal can be written as

$$
\mathbf{x}=\mathbf{W} \mathbf{s}=\sum_{k=1}^{K} P_{k} \mathbf{w}_{k} s_{k},
$$

where the individual precoder is $\mathbf{w} \in \mathbb{C}^{K}, P_{k}=\sqrt{\frac{P}{K}}$ is the equally transmitted power allocation and $s_{k} \in \mathbb{C}^{K}$ represents the data streams.

Then, the received signal of the $k$ th UE is given by

$$
y_{k}=P_{k} \mathbf{h}_{k}^{H} \mathbf{w}_{k} s_{k}+\sum_{j \neq k}^{K} P_{j} \mathbf{h}_{j}^{H} \mathbf{w}_{j} s_{j}+n_{k},
$$

And the SINR for UE $k$ can be analytically expressed as follows:

$$
\gamma_{k}=\frac{P_{k}\left|\mathbf{h}_{k}^{H} \mathbf{w}_{k}\right|^{2}}{\sum_{j \neq k}^{K} P_{j}\left|\mathbf{h}_{j}^{H} \mathbf{w}_{j}\right|^{2}+1}
$$

The work in [9] proposes a comparison between the three following schemes: (i) only macrocell, (ii) 
macrocell-picocell, and (iii) VSC. The network parameters are based on third Generation Partnership Project (3GPP) Long Term Evolution (LTE) (release 9) Technical Report 36.814 [22] and summarized in Table 1.

Also from [22] antenna pattern is computed based on the 3D model as $A(\theta, \varphi)=-\min \left(-A_{h}(\varphi)+A_{v}(\theta), A_{m}\right)$, with maximum attenuation $A_{m}$, where

$$
A_{v}(\theta)=-\min \left(12\left(\frac{\theta_{\mathrm{tilt}}}{\theta_{3 \mathrm{~dB}}}\right)^{2}, A_{m}\right)
$$

Table 1 System level simulation parameters for urban scenario

\begin{tabular}{|c|c|}
\hline Parameter & Value \\
\hline Handover margin & $1 \mathrm{~dB}$ \\
\hline Channel model & $3 \mathrm{GPP}$ macro, $3 \mathrm{~km} / \mathrm{h}$ \\
\hline Interference modeling & Explicit modeled \\
\hline Traffic model & Full buffer \\
\hline Number of UEs per sector & $20,100 \%$ outdoor \\
\hline \multirow[t]{4}{*}{ Antenna configuration } & Macrocell antenna gain $14 \mathrm{dBi}$ \\
\hline & $N_{\mathrm{mc}} T \times \mathrm{X}$-pol for macro sectors \\
\hline & Small cell antenna gain $0 \mathrm{dBi}$ \\
\hline & 1 Tx for physical small cell \\
\hline \multirow[t]{2}{*}{ Scheduler } & Proportional fair \\
\hline & 1 max number of co-scheduled UEs \\
\hline \multirow[t]{3}{*}{ MIMO scheme } & SU-MIMO $2 \times 2$ closed loop \\
\hline & Max rank: 2 \\
\hline & LTE precoding (release 8) \\
\hline \multirow[t]{4}{*}{ UE antenna } & Omni directional \\
\hline & Gain $0 \mathrm{dBi}$ \\
\hline & $2 R \times X-p o l$ \\
\hline & $1.5 \mathrm{~m}$ height \\
\hline UE receiver & MMSE \\
\hline UE noise figure & $9 \mathrm{~dB}$ \\
\hline Tiers & 3 \\
\hline Bandwidth & $10 \mathrm{MHz}$ \\
\hline Carrier $(\mathrm{MHz})$ & FDD DL - 2000 \\
\hline ISD (m) & 500 \\
\hline Hotspot radius (m) & 40 \\
\hline Av. building height (m) & 20 \\
\hline Penetration loss (dBm) & 20 \\
\hline Macrocell tx power (dBm) & 46 \\
\hline Power split between & $50 / 50 \%$ \\
\hline \multicolumn{2}{|l|}{ coverage beam/NSC beam } \\
\hline Picocell tx power (dBm) & 30 \\
\hline
\end{tabular}

for the vertical antenna pattern, and for the horizontal one

$$
A_{h}(\varphi)=-\min \left(12\left(\frac{\varphi_{\mathrm{tilt}}}{\varphi_{3 \mathrm{~dB}}}\right)^{2}, A_{m}\right)
$$

and the pathloss models used for the scenario "macrocell to UE" for non-line of sight (NLOS) is the one defined in the 2D 3GPP model:

$$
\mathrm{PL}=131.1+42.8 \log _{10}(R)
$$

where $R^{2}=$ dist $^{2}+h^{2}$ in kilometer from Fig. 2 . And in the scenario "picocell to UE,"

$$
\mathrm{PL}=145.4+37.5 \log _{10}(R)
$$

We consider an already localized hotspot of $40 \mathrm{~m}$ radius where the number of antenna elements are directly deduced (from (5) and (6)) and $N_{\mathrm{mc}}=10$ antennas for the macrocell. LTE precoding (release 8 ) is used based on transmit beamforming concepts. The LTE specification defines a set of complex weighting matrices for combining the layers before transmission. Codebook selection provides some flexibility when attempting to improve and equalize the SINR at each receiver. We consider 20 UEs in outdoor location, where around $60 \%$ are inside the hotspot. The consideration of more UEs implies more positioning information and higher density, improving the performances of the VSCs. Please note that this scenario with 20 UEs is the one considered in the 3GPP Technical Report for evaluation 38.814 [22]. Anyhow, from operator point of view, 20 UEs communicating at the same time in full buffer represents a dense traffic scenario. A representation of the UEs repartition for the simulated scenario is presented in Fig. 4.

By using a $\mathrm{C}++$ internal system level simulator, also used in $[9,14,23]$, we observe that when implementing the VSC, almost $80 \%$ of the considered UEs are attached to the VSC in a macrocell-VSC scenario, against a total of $60 \%$ of UEs inside the picocell coverage for a typical macrocell-picocell scenario. This is because the VSC covers not only the UEs within the hotspot but also some that are close to it. In that sense, decreasing the percentage of UEs into a forming hotspot with same size will decrease the density, and as a consequence, degrade the performances of the VSC identification. In other words, density is a key condition for VSCs implementation process. SINRs of three scenarios, i.e., (i) only macrocell, (ii) macrocell-picocell, and (iii) macrocell-VSC scenarios, have been studied and plotted in Fig. 5. The figure shows that SINR in the macrocell-VSC scheme follows the performance of the picocell scheme in $60 \%$ of the cases, and is as good as the only macrocell scheme for the rest while avoiding the deployment cost of picocells. A green approach on the macro site 3 BS LTE using picocells has been studied in [24] and a power consumption study for 


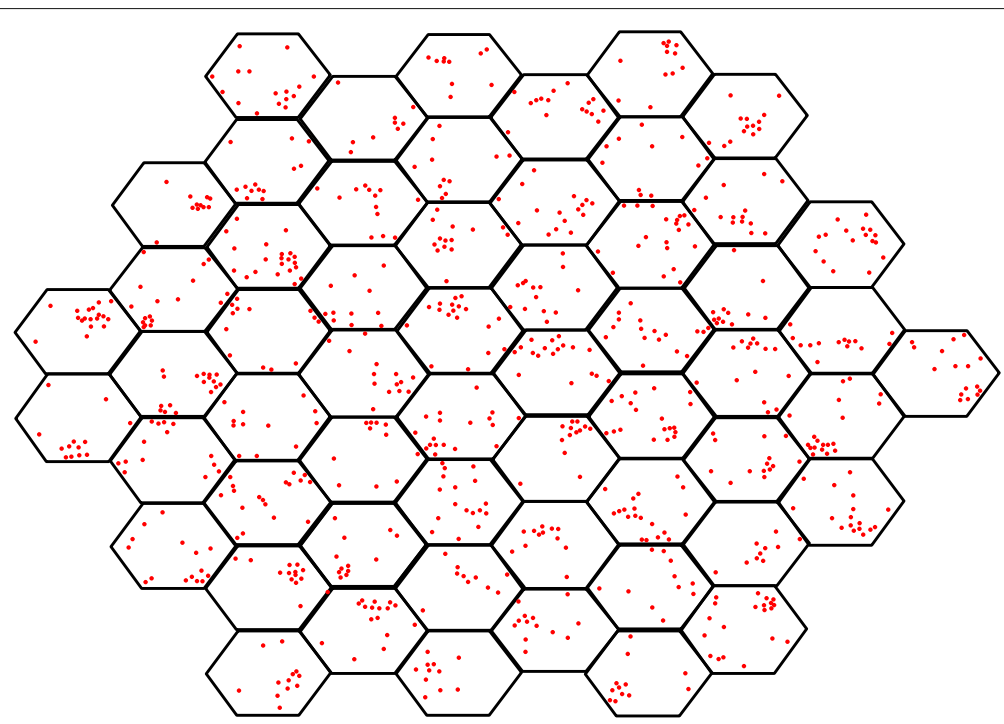

Fig. 4 UEs distribution in the network. This figure represents the UEs repartition in the network for the simulated scenario in Section 3

the proposed system is provided in [9]. Also a beamforming design minimizing the total power while maintaining throughput rate requirement is proposed in [10].

\section{Virtual small cell extended to several groups}

In really dense networks, there may be several hotspots physically separated and the need of implementing several VSCs in parallel. As we have seen in the previous section, the number of antennas depend on the size and orientation of the located hotspot. The more hotspots we have to

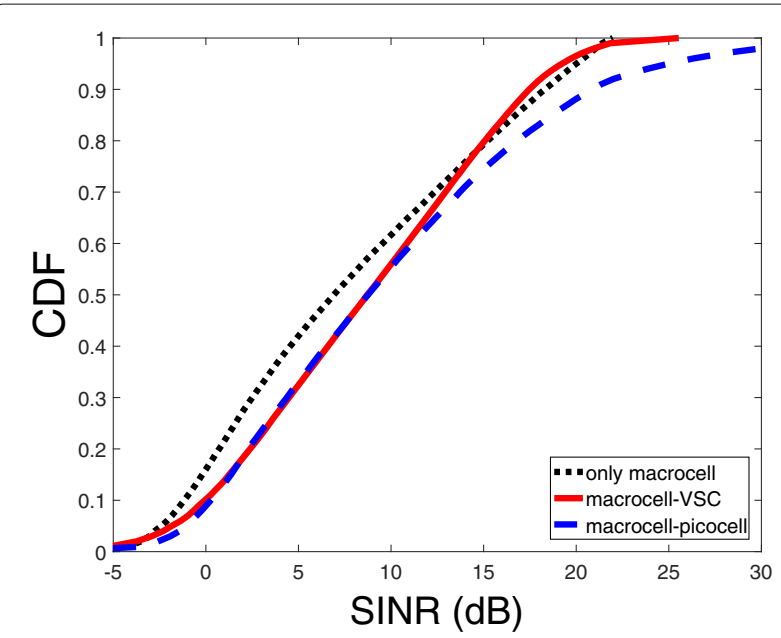

Fig. 5 SINR CDF at dense urban scenario [9]. This figure represents the SINRs of three scenarios, i.e., only macrocell, macrocell-VSC, and macrocell-picocell scenarios. Figure shows that SINR in the macrocell-VSC scheme follows the performance of the picocell scheme in $60 \%$ of the cases, and is as good as the only macrocell scheme for the rest while avoiding the deployment cost of picocells deal with, the more antennas at the BS we need. Figure 6 illustrates the deployment of the VSCs (Fig. 6b) that could replace the physical small cells (Fig. 6a), avoiding significant deployment cost for the operator.

\subsection{System model}

Let us consider the case where several VSCs could coexist, $M$ antennas at the BS serving $K$ UEs with $K \leq M$ thanks to massive MIMO. The UEs are still working in FDD over a Rayleigh-fading channel. The received signal and UEs channel are still represented by Eq. (8) where the dimension $M$ is much larger.

Work in [3] shows that human activity tends to be spatially clustered. In the literature, one of the most famous scheme for grouping is the joint spatial division multiplexing [8]. This scheme exploits an appropriate partitioning of the UEs such that those in the same group are collocated and then share the same UE correlation matrix defined by the one-ring model. This will reduce the dimensionality of the effective channels and therefore achieve large multiplexing gains with reduced dimension channel training and transmitter's CSI feedback. We make the assumption that $K$ UEs are partitioned into $G$ groups (or $G$ potential VSCs) where $K_{g}$ denotes the number of UEs in group $g$, not necessarily collocated, such that $\sum_{g=1}^{G} K_{g}=K$. The downlink channel of the general system can be written like

$$
\mathbf{H}=\left[\mathbf{H}_{1}, \ldots, \mathbf{H}_{G}\right]
$$

where $\mathbf{H}_{g}$ is the downlink channel of the gth group. By extending the one-ring model to the notion of VSC, as shown in Fig. 7, we can define the VSCs correlation matrix 

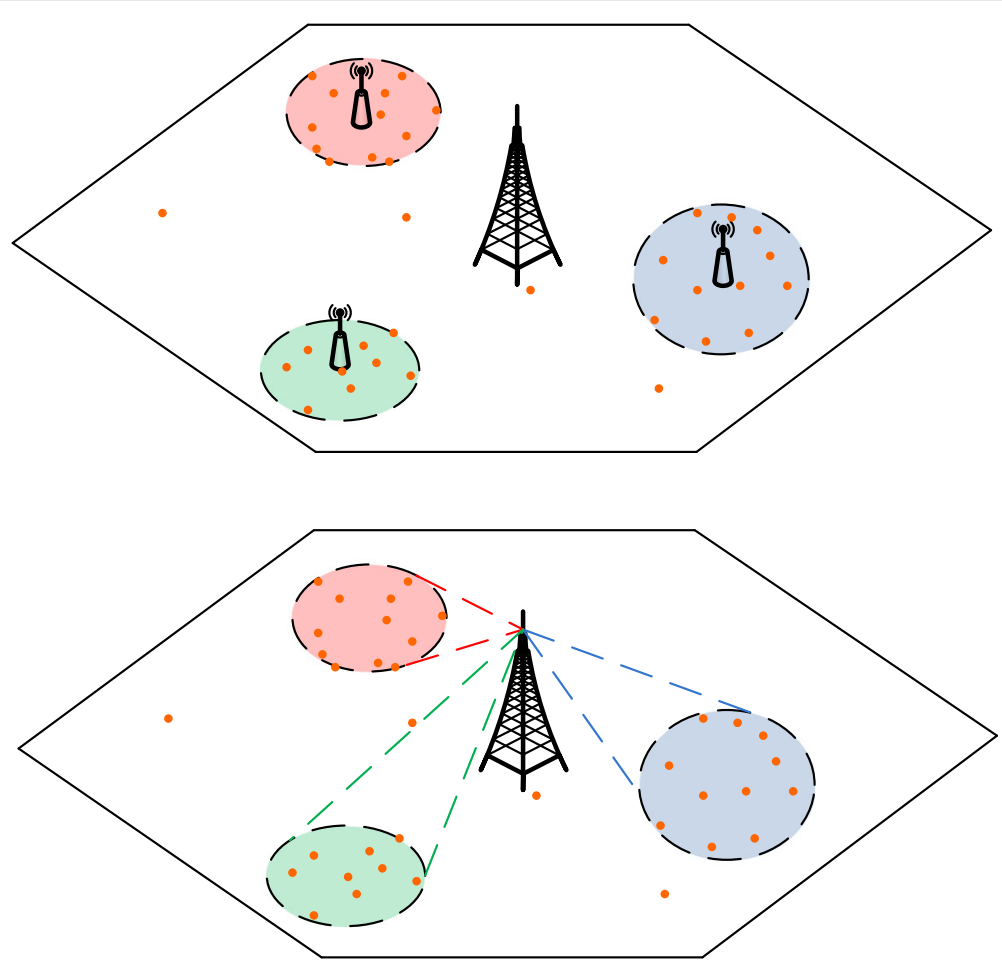

Fig. 6 Network topology with and without VSCs example. This figure illustrates the deployment of the VSCs (Fig. 4b Macrocell-VSCs scheme.) that could replace the physical small cells (Fig. 4a Macrocell-picocell scheme.), avoiding significant deployment cost for the operator

$\mathbf{R}_{g}$ corresponding to the $g$ th group covered by the $g$ th VSC in outdoor environment as

$$
\left[\mathbf{R}_{g}\right]_{i, j}=\frac{1}{2 \Delta \varphi_{3 \mathrm{~dB}_{g}}} \int_{\varphi_{\mathrm{tiltg}}-\Delta \varphi_{3 \mathrm{dBg}}}^{\varphi_{\mathrm{tiltg}}+\Delta \varphi_{3 \mathrm{dBg}}} e^{-j 2 \pi D(i-j) \sin (\alpha)} \mathrm{d} \alpha,
$$

for $0 \leq i, j \leq M-1$, that are the index of the antenna position in the array and $\lambda D$ is the minimum distance between two antenna elements at the BS. In addition, please note that our model differs from [8] as we consider a group correlation matrix, where UEs are not necessarily collocated, whereas in [8] authors consider that each collocated UE have the same correlation matrix.

To recall, when using the geometrical one-ring model, we consider a $120^{\circ}$ sector, obtained by using directional radiating elements, assuming that the sector is centered around the $x$ axis ( $\alpha=0$ azimuth angle), and that no energy is received for angles $\alpha \notin[-\pi / 3, \pi / 3]$. Also, uniform linear array is supposed, so that the covariance matrix $\mathbf{R}_{g}$ of the channel for a group $g$, with angle of arrival (AoA) $\varphi_{\text {tiltg }}$ and with angular spread of departure $\Delta \varphi_{3 \mathrm{~dB}_{g}}=\frac{\varphi_{3 d B_{g}}}{2}$ to group $g$, can be used in (17).

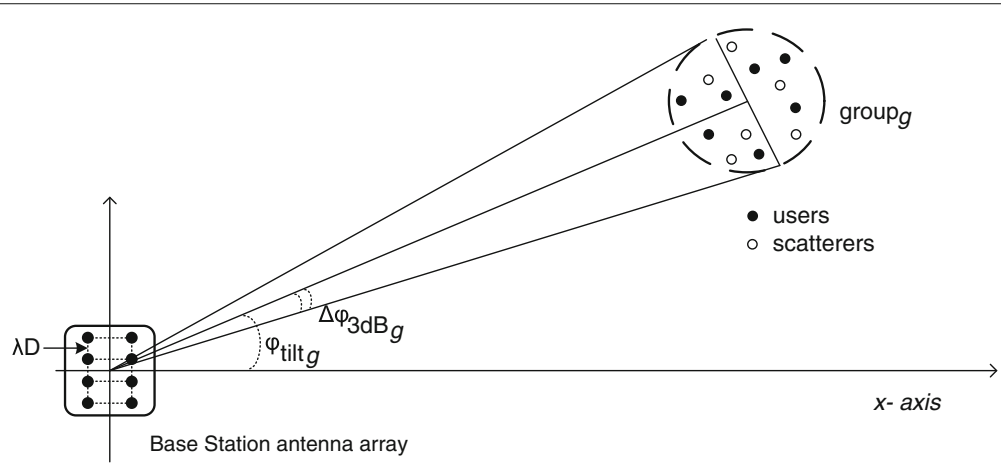

Fig. 7 Geometrical one-ring model extended to a group. This figure represents the correlation between the VSC and the one-ring model for correlation matrix 
The downlink channel of the $g$ th group is expressed by applying the Karhunen-Loeve model, as

$$
\mathbf{H}_{g}=\left[\mathbf{h}_{g 1}, \ldots, \mathbf{h}_{g K_{g}}\right]=\mathbf{U}_{g} \mathbf{\Lambda}_{g}^{\frac{1}{2}} \mathbf{G}_{g}
$$

with $\boldsymbol{\Lambda}_{g}=\operatorname{diag}\left(\operatorname{eig}\left(\mathbf{R}_{g}\right)\right) \in \mathbb{C}^{r_{g} \times r_{g}}$ which represents the diagonal matrix containing the non-zero eigenvalues of $\mathbf{R}_{g}$, where $\mathbf{R}_{g}$ is of rank $r_{g} . \mathbf{U}_{g} \in \mathbb{C}^{M \times r_{g}}$ is the associated eigenvectors matrix. The elements of $\mathbf{G}_{g} \in \mathbb{C}^{r_{g} \times K_{g}}$ are distributed according to $\mathcal{N}_{\mathcal{C}}(0,1)$. Also from (18), we can observe that the knowledge of $\mathbf{R}_{g}$ is enough to characterized $\mathbf{H}_{g}$. This highlights the relationship between the correlation matrix and the channel matrix for users belonging to group $g$.

Then, as in $[8,18]$, we use a two-tier precoder (TTP) scheme for FDD massive MIMO systems, and the transmitted signal is expressed as

$$
\mathbf{x}=\sum_{g=1}^{G} \mathbf{B}_{g} \mathbf{W}_{g} \mathbf{P}_{g} \mathbf{s}_{g},
$$

where $\mathbf{B}_{g} \in \mathbb{C}^{M \times b_{g}}$ represents the outer precoder for the groups, or pre-Beamforming matrix while the inner precoder, or individual precoder is $\mathbf{W}_{g} \in \mathbb{C}^{b_{g} \times K_{g}}$ and depends on the short-term effective channel $\tilde{\mathbf{H}}_{g}=\mathbf{B}_{g}^{H} \mathbf{H}_{g} . b_{g}$ is an integer design parameter, $\mathbf{P}_{g} \in \mathbb{C}^{K_{g} \times K_{g}}$ is the diagonal power allocation matrix $\sqrt{P / K} \times \mathbf{I}$ where $\mathbf{I}$ is the identity matrix and $\mathbf{s}_{g} \in \mathbb{C}^{K_{g}}$ represents the data streams for the $g$ th group UEs.

Then, in comparison with the case where there was only one VSC (10) and (11), the received signal $y_{g k}$ and the SINR $\gamma_{g k}$ of the $k$ th UE in the $g$ th group are respectively given by

$$
\begin{aligned}
& y_{g k}=P_{g k} \mathbf{h}_{g k}^{H} \mathbf{B}_{g} \mathbf{w}_{g k} s_{g k}+\sum_{j \neq k}^{K_{g}} P_{g j} \mathbf{h}_{g j}^{H} \mathbf{B}_{g} \mathbf{w}_{g j} s_{g j} \\
& +\sum_{l \neq g}^{G} \mathbf{h}_{g l}^{H} \mathbf{B}_{l} \mathbf{W}_{l} \mathbf{P}_{l} \mathbf{s}_{l}+n_{g k}, \\
& \gamma_{g k}=\frac{P_{g k}\left|\mathbf{h}_{g k}^{H} \mathbf{B}_{g} \mathbf{w}_{g k}\right|^{2}}{\sum_{j \neq k}^{K_{g}} P_{g j}\left|\mathbf{h}_{g j}^{H} \mathbf{B}_{g} \mathbf{w}_{g j}\right|^{2}+\sum_{l \neq g}^{G}\left|\mathbf{h}_{g l}^{H} \mathbf{B}_{l} \mathbf{W}_{l} \mathbf{P}_{l}\right|^{2}+1}
\end{aligned}
$$

\subsection{Codebook and feedback scheme}

In FDD, the feedback problem is complex as the downlink and uplink do not share the same band, hence, no reciprocity could be used. A classical way is to estimate the downlink channel from a training phase and then fed back to the BS via uplink signaling. This scheme could introduce feedback overhead in case of large amount of antenna at the BS as it is the case in massive MIMO where the downlink training represents a significant amount of information. This feedback overhead will degrade the benefits of massive MIMO. Also a new feedback scheme has been introduced by Verizon where the BS periodically transmits beams at different angles by transmitting beam reference signal, also known as beam sweeping. UE maintains a candidate beam set and report the one with best beam reference signal received power [25]. We could extend beam sweeping to group of UEs and then perform the VSC. Another way is to consider a predefined codebook that is available at the UE and at the BS. As we are working in FDD, and in order to reduce the feedback overhead, we will provide a grouping feedback scheme.

As it can be seen in (20), the outer precoder $\mathbf{B}_{g}$ is in charge of the grouping interference. This means that by effectively choosing this outer precoder, it can help canceling the effects of grouping interference. Information on the grouping downlink channel is needed for good outer precoder selection. As expressed in (18), correlation matrix $\mathbf{R}_{g}$ is enough to learn the downlink channel of the $g$ th group, and $\mathbf{R}_{g}$ depends on the angle information given by the knowledge of the hotspots location. By knowing the $g$ th hotspot center location, its radius and the BS location, we can compute the correlation matrix $\mathbf{R}_{g}$ and accordingly choose the outer precoder $\mathbf{B}_{g}$ in order to avoid group interference.

Those previous inputs enable us to compute $\varphi_{\text {tilt }}$ and $\Delta \varphi_{3 \mathrm{~dB}}=\frac{\varphi_{3 \mathrm{~dB}}}{2}$ corresponding to the detected hotspot. We consider the following predefined codebook:

$$
\mathcal{C}=\left\{\left(\varphi_{\text {tilt }}, \Delta \varphi_{3 \mathrm{~dB}}\right)_{i}, i=1,2, \ldots, 2^{B}\right\}
$$

The values of the angles are compared with those in the codebook, where angles matching gives us the corresponding index $i$. While this index is known by the BS, whether because it has been given by a specific UE belonging to the hotspot or because it has been computed by the BS itself, we can select the pre-computed correlation matrix corresponding to the couple angles given by index $i$ and then the outer precoder. The BS is now aware of the best precoding intended for this group and will apply it in order to focus the directive beam on the detected hotspot.

For the individual precoder $\mathbf{W}_{g}$, also called inner precoder, we can apply the same procedure as in Section 3 and consider only one hotspot or we can use the methodology given by [26]. There the UEs benefit from D2D technology and exchange their CSI such that a cluster head $(\mathrm{CH})$ is aware of the global CSI and select the adequate precoder for each UE.

Now the problem is how to detect the hotspots corresponding to the groups in order to find the angles $\varphi_{3 \mathrm{~dB}_{g}}$ and $\varphi_{\text {tilt }}$ for the correlation matrix $\mathbf{R}_{g}$ such that the VSCs can be focused on the good direction. 


\section{Group localization}

To be able to implement the VSCs, we have to characterize their center and their radius. In this section, we aim to develop algorithms to identify these two values depending on the operator strategy. Therefore, our algorithm is able to automatically detect hotspots and dynamically identify a variation of these two characteristics allowing the network to adapt in real time its coverage.

\subsection{UEs mapping using Global Positioning System}

Here we respond to the missing point of hotspot location by proposing a dynamic clustering algorithm based on Global Positioning System (GPS) coordinate location. We suppose that the BS benefits from minimization of drive tests (MDT) [27] which collects network quality information like UE coordinates, i.e., MDT geo-localize the UEs. Detailed location information provided by MDT reports, allows the operator to associate a set of MDT measurements with a physical location. The UEs are requested by the BS to acquire location information for a configured MDT session [28]. The knowledge of the UEs coordinates is hence available at the BS and can be used in order to find the hotspots. The BS will apply an algorithm based on the well known K-means that outputs or create clusters of UEs. Then, after some optimizations, we succeed on transforming the clusters into hotspots.

The general algorithm for hotspot detection is constructed as in [14]. First, K-means is an unsupervised learning algorithm that solves clustering problem. In Kmeans, a set of $K$ data points and an integer $C$ which represents the number of centers to evaluate are given. These centers are randomly defined and then optimized in order to minimize the mean squared distance from each data point to its nearest center [29], as described between the lines 1 and 15 in Algorithm 1. Then, the optimal number of clusters is selected based on the distortion function provided by [30], as explained in Algorithm 1 in lines 16 to 23. Secondly, to avoid UEs located far from the cluster and still grouped to it by K-means, a process that removes the far UEs is implemented, which is presented between lines 24 and 32. Also, please notice that if a cluster is considered as low populated it will be removed from the database, where $n$ is a parameter configurable by the operator. The last optimization is on determining if the cluster can be smaller covering almost the same number of UEs by looking each cluster density thanks to their distortion values. Parameter $p$ line 37 represents a fraction of the inter site distance.

In order to evaluate the potential of our algorithm, let us consider a system composed of 57 hexagonal cell with inter site distance (ISD) of $500 \mathrm{~m}$. Then, in each cell, we have "created" a circle of $40 \mathrm{~m}$ radius and randomly placed $60 \%$ of the UEs inside and $40 \%$ outside this circle, as represented in Fig. 4. We consider perfect GPS localization.

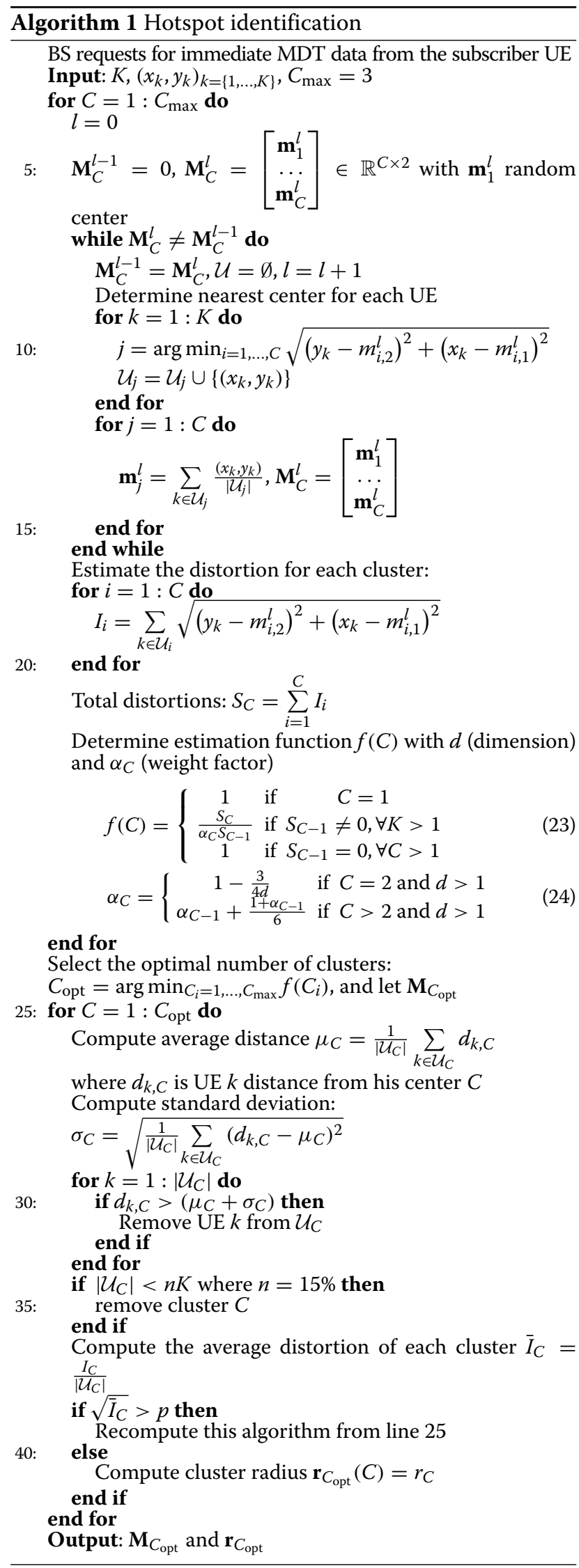


The error GPS localization will be taken into account in Section 6. When applying the proposed hotspot identification algorithm to each cell and by repeating the process 100 times, we obtain a probability of $55 \%$ of detecting one hotspot per sector, $41 \%$ of detecting two hotspots and $4 \%$ of detecting three hotspots. This means that new groups of UEs have been detected. Also, the hotspot identification algorithm is covering $72 \%$ of the total population which is $12 \%$ more than the considered cellular system. Furthermore, after repeating the process hundred times, half of the radius measured are between 30 and $45 \mathrm{~m}$, as it can be seen in Fig. 8.

In Fig. 9, 25\% of the radius are below $32.7 \mathrm{~m}$, half of the radius are below $39.4 \mathrm{~m}$ and $75 \%$ of the radius are below $50 \mathrm{~m}$. A nearly Gaussian distribution for the cluster radius is observed which means that the closest value from the mean are more able to appear than the others. This is satisfactory as half of the clusters radius are close to $40 \mathrm{~m}$.

Now that the BS is aware of the center location and radius of the detected hotspot, the couple $\left(\varphi_{\text {tilt }}, \Delta_{\varphi_{3 \mathrm{~dB}}}\right)$ can be computed thanks to (3) and (4), then a comparison is made between this couple of angles and the ones in the predefined codebook $\mathcal{C}$ in (22). The index $i$, corresponding to the closest couple in $\mathcal{C}$ with respect to the calculated one, is chosen and gives the pre-computed correlation matrix corresponding to index $i$ and then the outer precoder is deduce as explained in Section 4.2.

However, depending on the operator strategy, it might be more interesting to have a centralized hotspot localization at the UE side such that the BS is not overcharged by the UEs data. Furthermore, GPS provides a meter accuracy, which might be non-sufficient. In the next subsection, UEs are localized based on radio links.

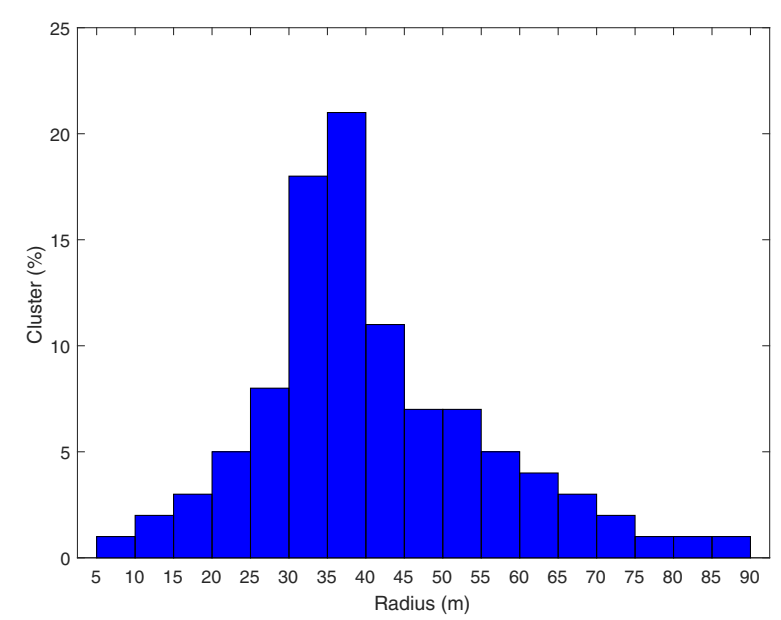

Fig. 8 Clusters radius distribution. This histogram represents the results on an analysis on a hundred of iterations that have been performed and half of the radius measured are between 30 and $45 \mathrm{~m}$

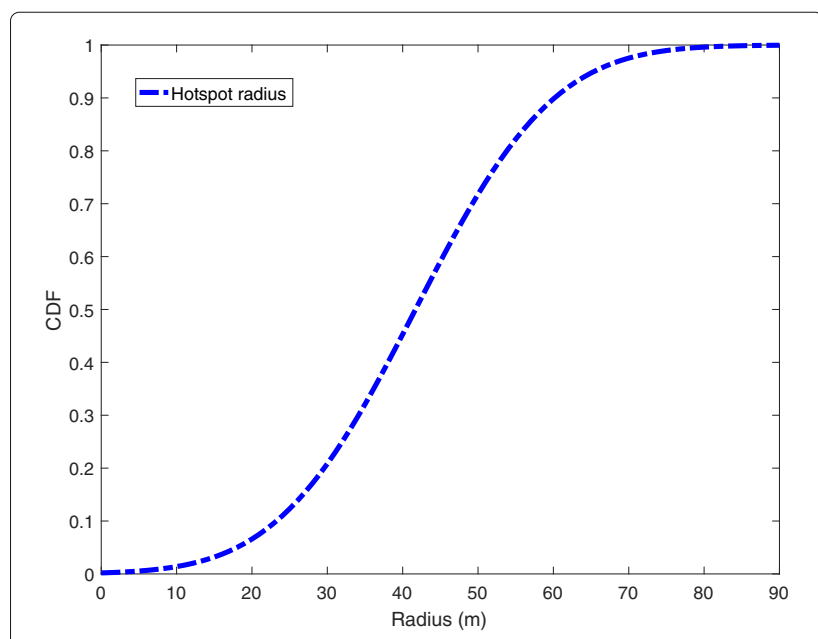

Fig. 9 Statistics on hotspots radius. This figure represents a CDF of the hotspots radius where $25 \%$ of the radius are below $32.7 \mathrm{~m}$, half of the radius are below $39.4 \mathrm{~m}$ and $75 \%$ of the radius are below $50 \mathrm{~m}$. A nearly Gaussian distribution for the cluster radius is observed which means that the closest value from the mean are more able to appear than the others

\subsection{UE mapping using direct radio links}

In this section, instead of studying a centralized positioning algorithm at the $\mathrm{BS}$, as presented in the previous subsection, we will provide a UE level algorithm using only distances between the UEs. In that sense, we suppose that all the UEs are able to directly communicate between each other, i.e., they have D2D communication capability, where this technology has been added to the 3GPP release 12 [31]. Usually, studies on D2D are about increasing the data rate [32] or extending coverage. It has also been proved that within a certain distance it is better to communicate through D2D than passing through the cellular BS [33]. In this paper, we would like to create a map of all the UEs by exchanging information in order to detect neighbors devices as in [34-36]. The information exchanged will be only distance parameters and no angles as in [34], as the axis of the mobile device depends on current orientation or altitude which implies that the angles are perpetually changing. Before going further, we are going to see how can the distances be measured between two devices without angle information and without anchor nodes, as in [35]. In order to detect the neighbor UE with highest possible accuracy, we propose to consider ultra-wideband (UWB) signals between the UE, which will help to provide D2D positioning. UWB is part of the IEEE 802.15 working group which specifies wireless personal area network (WPAN) standards [37]. In that sense, we may expect a future use of UWB for D2D communications.

Through its very large bandwidth, UWB radio link emits short low energy pulses and can achieve high immunity 
against interference and fading, unlike ultrasound or infrared. Furthermore, UWB has low energy consumption [38] and very high accuracy thanks to its wide frequency band [39] as it is indicated by Cramer-Rao lower band (CRLB) [40]. A disadvantage of UWB is its coverage range which can be solved thanks to a well organized routing topology between the smart devices, as explained in Section 5.2.3. From the ranging techniques perspective, time of arrival (ToA) is a one-way time difference between the moment the receiver detects the transmitters' signal and the time when the transmitter sends the signal. Usually, ToA offers a better accuracy than received signal strength and AoA for UWB positioning systems thanks to the high time resolution. Fortunately, due to the large bandwidth of UWB signal, multi-path components are often resolvable without the use of complex algorithms [41]. And due to existing high performance ToA estimation for UWB in NLOS [42-44], we will focus on this ranging technique. In order to work with ToA, we need to suppose perfect synchronization between the UEs.

Now that signal localization and ranging techniques are known, we will start the localization.

\subsubsection{Group mapping methodology}

First of all, a $\mathrm{CH}$ is chosen according to the desired strategy, e.g., in [45] authors select for each VSC a CH that will be in charge of connecting all the UEs belonging to the same VSC. In our paper, a $\mathrm{CH}$ has a specific processor capable of generating the treatment of the algorithm map, in order to represent the UEs in part of an area. The $\mathrm{CH}$ is also the first UE launching the algorithm procedure. As described in Fig. 10, first the $\mathrm{CH}$ will broadcast an information group message to the neighbors' UEs including a group ID, radio resources for transmission and reception of localization signal and also a threshold $P$ of power limit. Once an unlocalized UE, called blind UE, receives the information group message, if this UE is interested by joining the group, he will broadcast back a positive response to the UEs already localized by using the radio resources provided by the $\mathrm{CH}$. Evaluated distance between the transmitter and the receiver will be transmitted to the $\mathrm{CH}$. During the initialization step of the map, as we have a limited distance knowledge of the neighbor UEs, any blind UE is able to integrate our map as long as the threshold received power $P$ is satisfied. The initialization map is the smallest possible map that we are able to

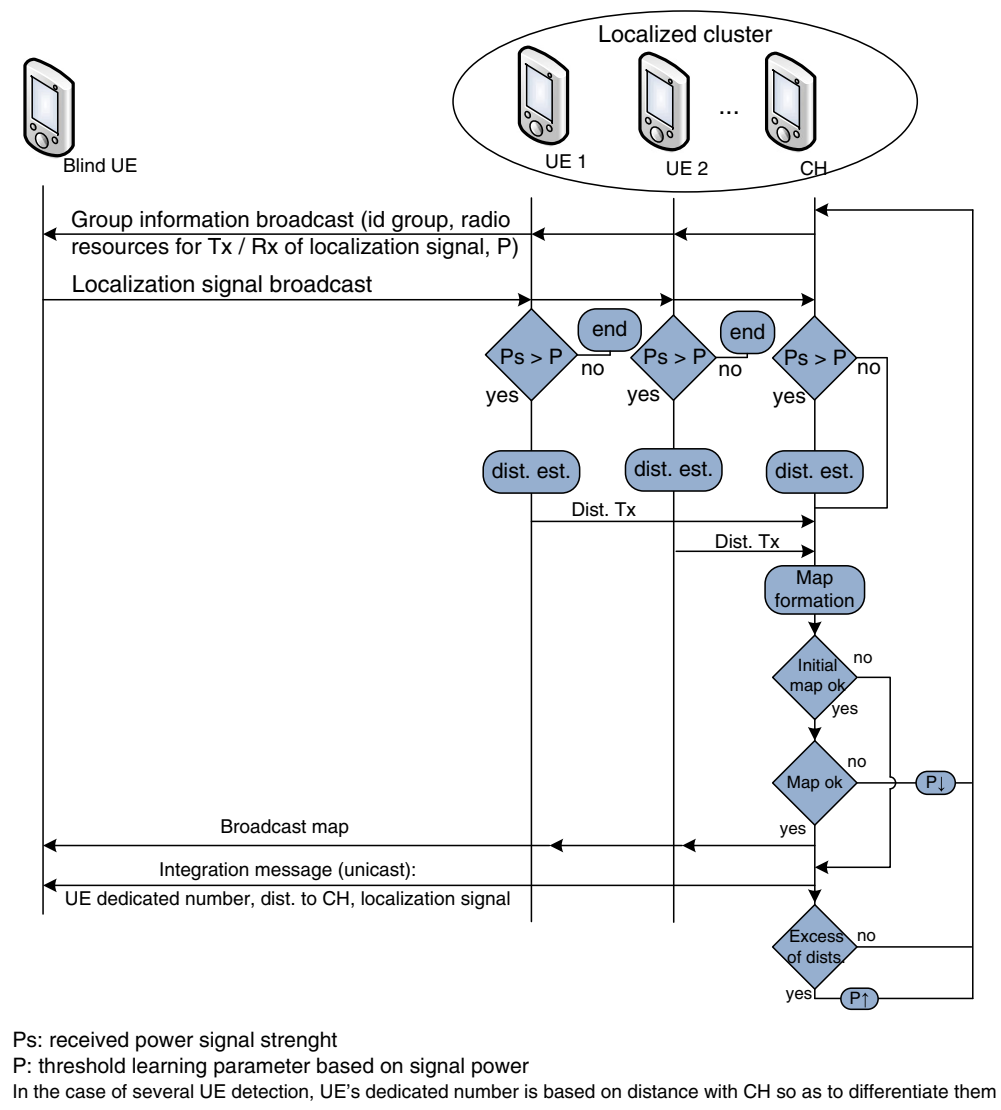

Fig. 10 General map steps. This figure represents the general diagram steps in order to create a map of UE 
form, and is composed of at least four UEs as stated in the Proposition 1 and proved in a previous work [16].

Proposition 1 With only $n=4$ UEs and the knowledge of $C_{n}^{2}=6$ distances named $d_{i j} \in \mathbb{R}$ between $U E i$ and $j\left(\forall\{i, j\}_{i=1, j>i}^{n}\right)$, we can map the $n$ UEs in an orthonormal coordinate system where the origin corresponds to the $\mathrm{CH}$ or any other UE.

$\mathrm{CH}$ is included within these four UEs. He is aware of the six distances between them, and each distance provides an equation such as

$$
d_{i j}=\sqrt{\left(y_{j}-y_{i}\right)^{2}+\left(x_{j}-x_{i}\right)^{2}}
$$

between UE $i$ and $j$, with four unknown values (each UE has two coordinates). We then have six equations of this type for the case of four UEs (eight unknown values). Actually, as the wanted map is relative to the $\mathrm{CH}$, we can consider that the $\mathrm{CH}$ coordinates are at the origin of the map and are $(0,0)$. So we finally have six equations for six unknown values, that consist of a solvable system of equations. Once the initial map is formed, this means that we have a map of at least four UEs. Then, when a blind UE $k$ wants to join the map, if an already localized UE receives a signal from the previous one with a received power $P_{k}<P$, the algorithm will not be launched as we consider only the reliable signals for distance estimation. Trilateration method is enough in order to add a blind UE $k$ to our map, as we need the distances between the new UE and three other UEs $(h, i$, and $j$ ) already localized. The blind UE $k$ is located at the intersection of the three circles of center $h, i$, and $j$ and radius $d_{h k}, d_{i k}$, and $d_{j k}$, respectively. The three distances used to localize the blind UE $k$ correspond to the three most accurate distances selected thanks to the received power. If the threshold power $P$ is too high such that the $\mathrm{CH}$ does not collect at least three signals, $P$ will be reduced at the next iteration. Finally, if a UE is added to the map, a broadcast of the map is possible and an integration message is sent by the $\mathrm{CH}$ to the new UE.

Proposition 2 With $n>4$, a map can be created with only $6+3(n-4)\left(<C_{n}^{2}\right)$ distances.

Proposition 2, provided in [16] means that we do not need to know all the distances between all the UEs in order to form a map. In order to add a blind UE to our map, we will need to solve a system of three equations such as (25). Also for good accuracy of the system, we will accept an incertitude of $+/-x$ meters in order to solve our system of equations. It will give output a location zone for blind UE called measurement incertitude, as represented in Fig. 11,

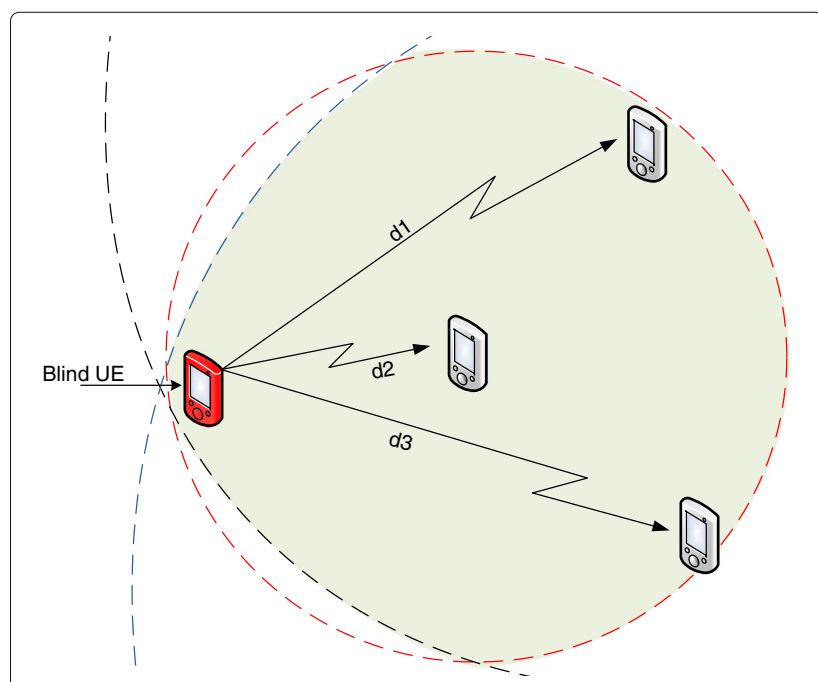

Fig. 11 Measurement incertitude model. This figure represents a location zone for blind UE called measurement incertitude zone, where the white UE are the already localized ones and the red one represents the blind UE

where the white UEs are the already localized ones and the red one represents the blind UE $k$.

If the system of equations provides no solution, i.e., no location zone, this means that one of the distance values does not match with the others, which may happen in case of strong error estimation of a distance. If this occurs, the $\mathrm{CH}$ will look for another distance, called redundant distance, possibly available at the $\mathrm{CH}$ as detailed in Corollary 1.

Corollary 1 In the case of mapping failure due to error distance estimation $(n>4)$, we benefit from additional $n-4$ redundant distances to create a map.

When adding UE $n$ into a map, we benefit from $n-1$ new possible distances. In order to add this new UE into the map, we only need additional $[6+3(n-4)]-[6+3(n-$ $1-4)]=3$ distances (i.e., based on Proposition 2). So we benefit from $n-1-3=n-4$ additional distances in case of mapping failure. The redundant distances are directly affected by the threshold $P$, as higher $P$ is, less redundant distances will be available. If no solution is provided by our system of equations, we reduce $P$. But even with such selection, the zone where blind UE $k$ is possibly localized is still inaccurate.

\subsubsection{Accuracy improvement}

In the previous section, the estimated blind UE position is normally the center of the zone, which might not be accurate enough as illustrated in Fig. 11. In this section, we will focus on improving the accuracy of the trilateration. 
Algorithm 2 needs as input parameters the set of the three best distances $\mathcal{D}$ between the blind UE and the group $\mathcal{U}$ of already localized UEs. The goal of the algorithm is to tight this zones, given by trilateration, by dividing it into sub zones and selecting the appropriated one. The procedure is based on dichotomy research where the value parameter for selection is represented by line 13 in Algorithm 2 . Each iteration gives output a more accurate zone where its center is selected as the estimated location of the blind UE. All these steps are detailed in Algorithm 2, and once we apply this algorithm to the previous measurement incertitude zone (Fig. 11), we see an improvement of the accuracy as our estimated position is closer to the real one (Fig. 12).

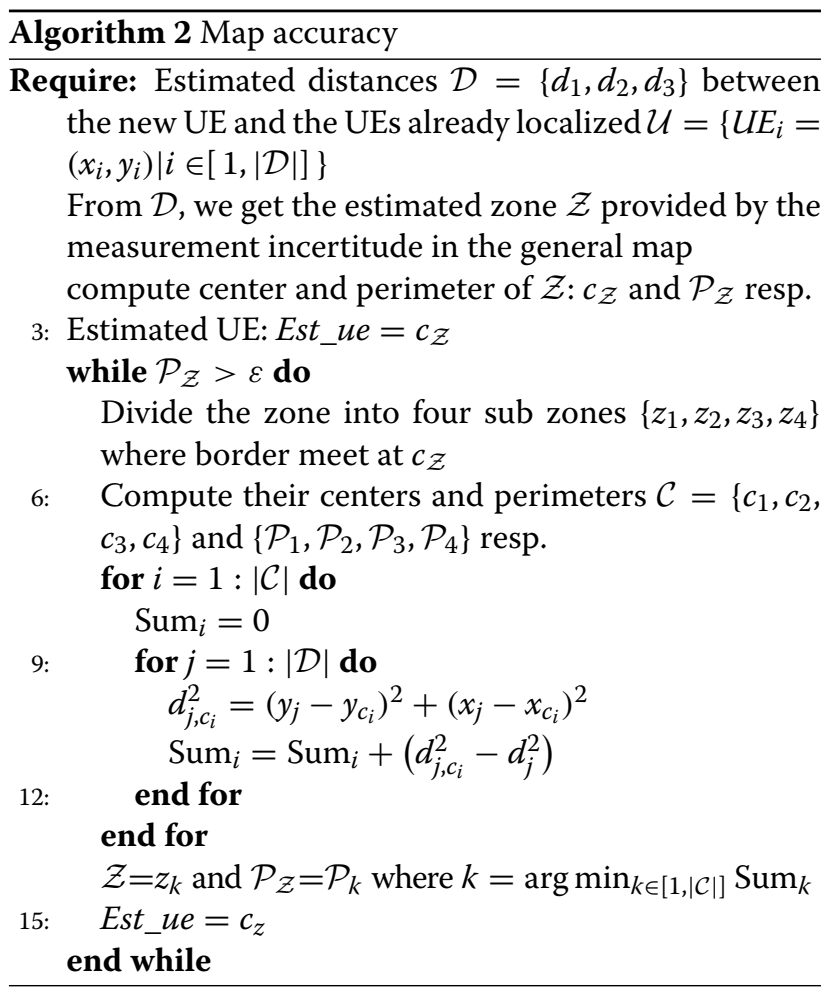

\subsubsection{UWB coverage extension and multi-group detection}

As the coverage of UWB is limited to around $20 \mathrm{~m}$ [46], this rang has to be increased in order to satisfy the VSCs coverage specifications which is around $40 \mathrm{~m}$, as the VSCs are supposed to replace the picocells. One obvious option is by relaying the UWB signals. Let us now introduce UEs that have relaying capabilities. Our network is hence composed of four type of UEs:

- The $\mathrm{CH}$, which is the one that launches the localization procedure and computes its corresponding map.

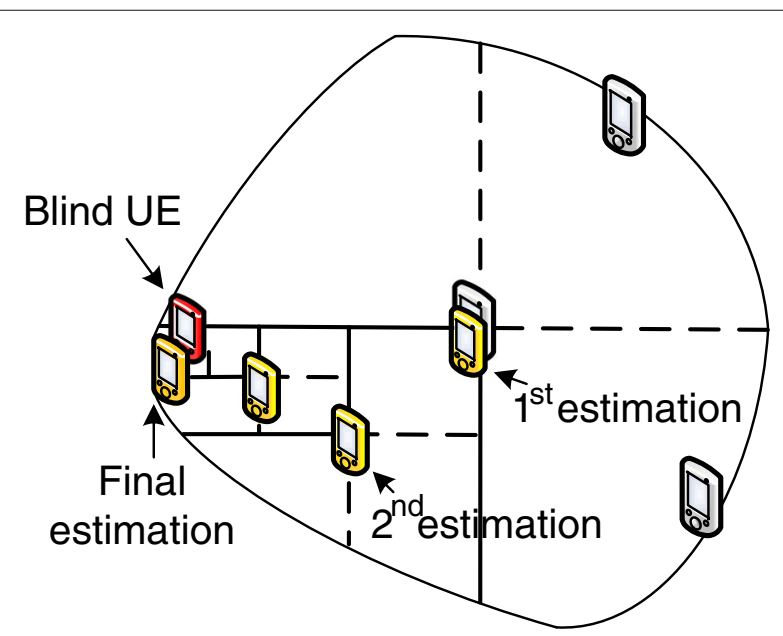

Fig. 12 Blind UE positioning accuracy applying Algorithm 2. This figure represents a location zone for blind UE after the optimization provide by Algorithm 2, where the yellow UEs are the estimated localization at each steps. The red one represents the blind UE

- The localized UE, which has been detected by a group. Its position is known by the $\mathrm{CH}$. Once a localized UE belongs to a group, it stops listening to others group information signals.

- The blind UE, which has not been localize by any group and is still listening to group information signals.

- The relaying UE, that is localized at the border of its corresponding groups and is still listening to group information signals. Its goal is to relay the broadcast localization signal send by the $\mathrm{CH}$ in order to extend the coverage. It is elected as relaying UE by any UE that is transmitting the group information broadcast signal, i.e., by a $\mathrm{CH}$ or any other relay.

We suppose that each UE is composed of a frame that is divided into three subframes, one for the group ID, one for the role of the UE (CH, relaying, localized, or blind UE), and one for the number of hops to the $\mathrm{CH}$.

As mentioned in Section 5.2.1, the $\mathrm{CH}$ is computing the localization map (see Fig. 10). Once the maximum UWB coverage rang is achieved and the map is performed, the $\mathrm{CH}$ will select the relaying UEs localized in the border of the group. All these UEs will be informed by the $\mathrm{CH}$ of their group ID, role (localized or relaying UE) and number of hops to the $\mathrm{CH}$. Then, the relaying UEs will extend the UWB coverage by retransmitting the group information broadcast signal intended to the still blind UEs and repeat the same procedure until no UEs are localized.

In the case that a relay from group $g$ detects a $\mathrm{CH}$ or a relay belonging to group $h$, this means that there may 
be one hotspot instead of two. In such a case, relay from group $g$ and $h$ will compare their corresponding hops subframe, and the one with less number of hops with its corresponding $\mathrm{CH}$ will change of group. Automatically, all the UEs attached to the relay that just made a group change will belong to the new group too. Their group ID subframe will change and the number of hops will be incremented. This is represented in Fig. 13, where $U E_{g i}$ and $U E_{h j}$ are two relays belonging to two different groups $g$ and $h$, respectively. One of these relays detect the other, and they compare their subframe corresponding to the number of hops to their respective $\mathrm{CH}$. Here, the number of hops of $U E_{g i}$ is 2 and the one of $U E_{h j}$ is 1 so group $h$ will be integrated into group $g$ as represented in the figure. Also, the number of hops in the subframe of $U E_{h j}$ is now 3 and the role of $\mathrm{CH}_{h}$ has changed into relaying $\mathrm{UE}$ and its corresponding number of hops is now 4 . Now that the coverage extension of UWB localization is no more a problem, we can apply part of the Algorithm 1 in order to transform the map of a group of UEs into a map of hotspot.

\subsubsection{Results for UE mapping using UWB signals}

We will now apply our UWB localization method and see how accurate it is in line of sight (LOS) and NLOS outdoor environment.

The channel model used below respects the IEEE 802.15.4a standards for UWB. As we are following the European regulation, we selected the best bandwidth $B$ which is $1 \mathrm{GHz}$ centered in $f_{c}=3993.6 \mathrm{MHz}$ where $f_{c}$ represents the carrier frequency [47]. We recall the pathloss model defined in IEEE 802.15.4a standards for UWB:

$$
\mathrm{PL}=\mathrm{PL}_{0}+10 n \log (d)
$$

where PL represent the pathloss, $\mathrm{PL}_{0}(\mathrm{~dB})$ and $n$ are specific values depending on the channel model. $d$ is the real distance between the transmitter and the receiver [46]. The signal to noise ratio (SNR) is expressed as

$$
\mathrm{SNR}_{\mathrm{dB}}=P_{t}-\mathrm{PL}-P_{n}
$$

where $P_{t}$ and $P_{n}$ are the transmitted and noisy power, respectively. From the standards [46], their power density are equal to $-41 \mathrm{dBm} / \mathrm{MHz}$ and $-174 \mathrm{dBm} / \mathrm{Hz}$, respectively. As our bandwidth is $B=1 \mathrm{GHz}, P_{t}=-41 \mathrm{~dB}$ and $P_{n}=-114 \mathrm{~dB}$. Because we use estimated distances as input, we focused on the CRLB distance error which is one of the most commonly used measure of ToA accuracy [40]. From [41], the accuracy of ToA can be improved by increasing the SNR or the effective signal bandwidth. Since UWB signals have very large bandwidths, we benefit from extremely accurate location estimation using ToA via UWB radios. Also an approximation method of the CRLB when using UWB signals is introduced in [48] and an other one using millimeter-wave (mm-wave) is proposed in [49]. The distance error, $d_{\mathrm{CRLB}}$, is given by the CRLB and the estimated distance is $d+d_{\text {CRLB }}$, where

$$
d_{\mathrm{CRLB}} \approx \frac{c}{2 \pi B \sqrt{\mathrm{SNR} \text { Watt }}}
$$

The measurements were done following the IEEE 802.15.4a standards for LOS and NLOS in outdoors [46].

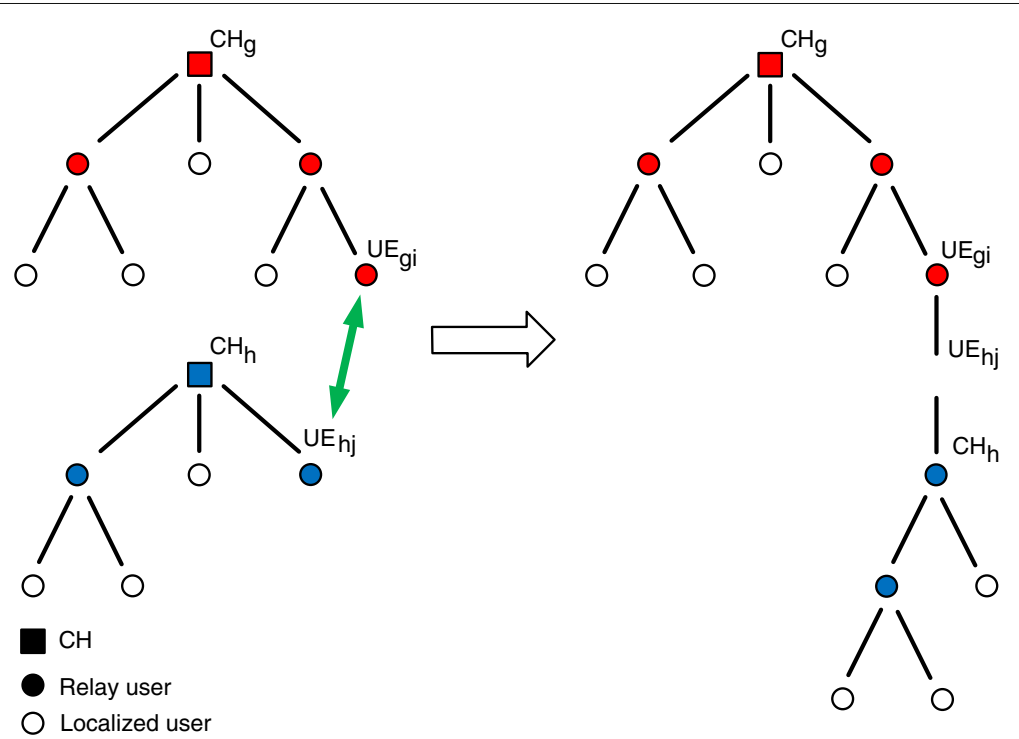

Fig. $13 U W B$ coverage extension. This figure represents the case where a relay from group $g$ detects a relay belonging to group $h$. This means that there may be one hotspot instead of two. In such a case, relay from group $g$ and $h$ will compare their hops with their corresponding $\mathrm{CH}$, and the one with less number of hops will change group. Automatically, all the UEs attached to the relay that just made a group change will belong to the new group too 
Figure 14 presents the repartition depending on the error between the blind UE position and the estimated position for LOS and NLOS outdoor. These results are obtained after 100 simulations. The simulation results show that the localization accuracy in LOS environments has an estimation error below $2 \mathrm{~cm}$ in $73 \%$ of the cases and below $55 \mathrm{~cm}$ in $71 \%$ of the case in NLOS environments. Please notice that half of errors estimation are below $1 \mathrm{~cm}$ in LOS. Also, for LOS static UEs, [50] considered the performance of minimum mean squared error (MMSE) localization, where the difference between the true position and the
MMSE estimate position is presented in their Fig. 14. Major part of their estimations are around $3 \mathrm{~cm}$ accuracy. In the next section, we compare our results to GPS approach used in our first group localization method in Section 5.1 whose error localization will be considered and is around $10 \mathrm{~m}$ in LOS outdoor environment [51]; as GPS remains the notorious outdoor localization technique. Meanwhile, we propose a solution for the step after ToA estimations, several works focus on resolving the most common problems of ToA signals [52-55]. Please notice that we can achieve even better results with our
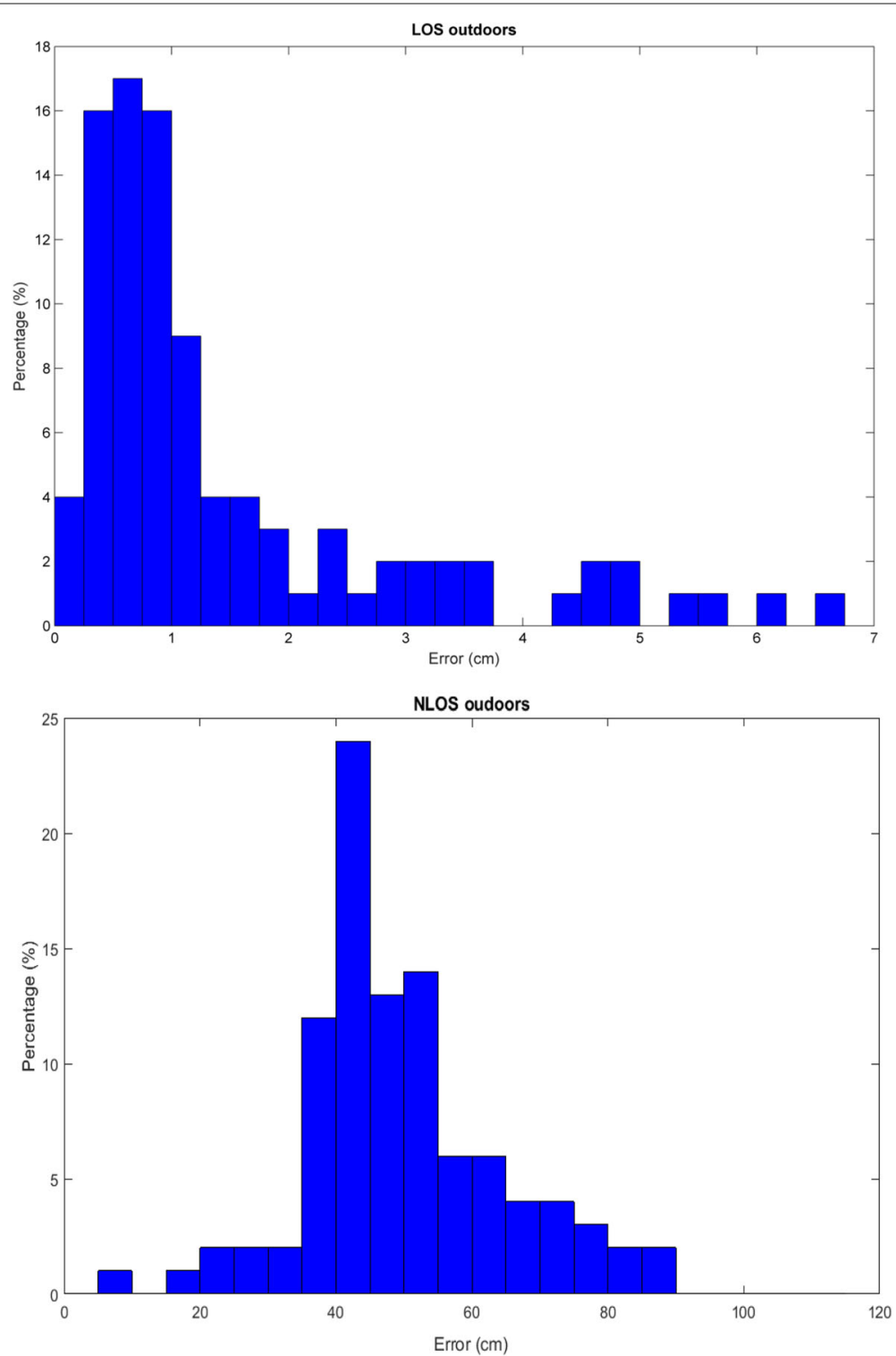

Fig. 14 Algorithm simulations for LOS and NLOS outdoor UWB channel model. This figure represents the repartition depending on the error between the blind UE position and the estimated position for LOS and NLOS outdoor. These results are obtained after 100 simulations. The simulation results show that the localization accuracy in LOS environments has an estimation error below $2 \mathrm{~cm}$ in $73 \%$ of the cases and below $55 \mathrm{~cm}$ in $71 \%$ of the case in NLOS environments 
algorithm while combining these two visions, i.e., use efficient ToA as input to our algorithm. The $\mathrm{CHs}$ have now the maps of the hotspots from its point of view, i.e., from the orthonormal coordinate system where it is located at the origin.

Remark 1 When creating such a map, we observe infinite solutions depending on the orthonormal coordinate system, but all the solutions will provide the same map with some rotation between 0 and $2 \pi$. Uniqueness of the map can be achieved by fixing the coordinate system, i.e., by the knowledge of the cellular coordinate of one UE (different than the $\mathrm{CH}$ ).

So, supposing that the $\mathrm{CH}$ is aware of its cellular coordinates and also the ones of another UE in its map, it will be able to compute the center location and radius of the mapped hotspot. The couple $\left(\varphi_{\text {tilt }}, \Delta_{\varphi_{3 \mathrm{~dB}}}\right)$ are deduce from (3) and (4), then a comparison is made between this couple and the ones in the predefined $\operatorname{codebook} \mathcal{C}$ in (22), available at $\mathrm{CH}$ side. The index $i$, corresponding to the closest couple in $\mathcal{C}$ with respect to the calculated one, is chosen and fed back to the BS. As only the $\mathrm{CH}$ is feeding back to the BS and not all the UEs, this will avoid feedback overhead for the case of large amount of transmit antennas. The BS can now choose the pre-computed correlation matrix corresponding to index $i$ and then the outer precoder is deduced and applied in order to focus the directive beams on the detected hotspots.

\section{Results and discussion}

As our hotspot localization methods are intended for the use of the VSCs, we consider a cellular system with 150 UEs distributed in such a way that in each sector $60 \%$ of the UEs are clustered in a $40 \mathrm{~m}$ hotspot radius and the rest are randomly distributed (i.e., like if there was a picocell in a cellular sector). We apply our two hotspot localization methods for 100 simulations and have highlighted four representative clustering realizations depending on UEs distribution, as shown in Fig. 15. In realization $i$, presented in Fig. 15a, both methods give us a hotspot where the one given by UWB signals has a smaller radius than the one given by the GPS, in particular, due to the GPS error localization of $10 \mathrm{~m}$ in average [51]. Figure 15b highlight a second representative realization where the $40 \%$ of the UEs, that are outside the predefined hotspot, are distributed in such a way that they form a second hotspot in the cellular sector. Meanwhile, both clustering methods detect this hotspot, its radius is much smaller using UWB method than GPS and also seems to be more
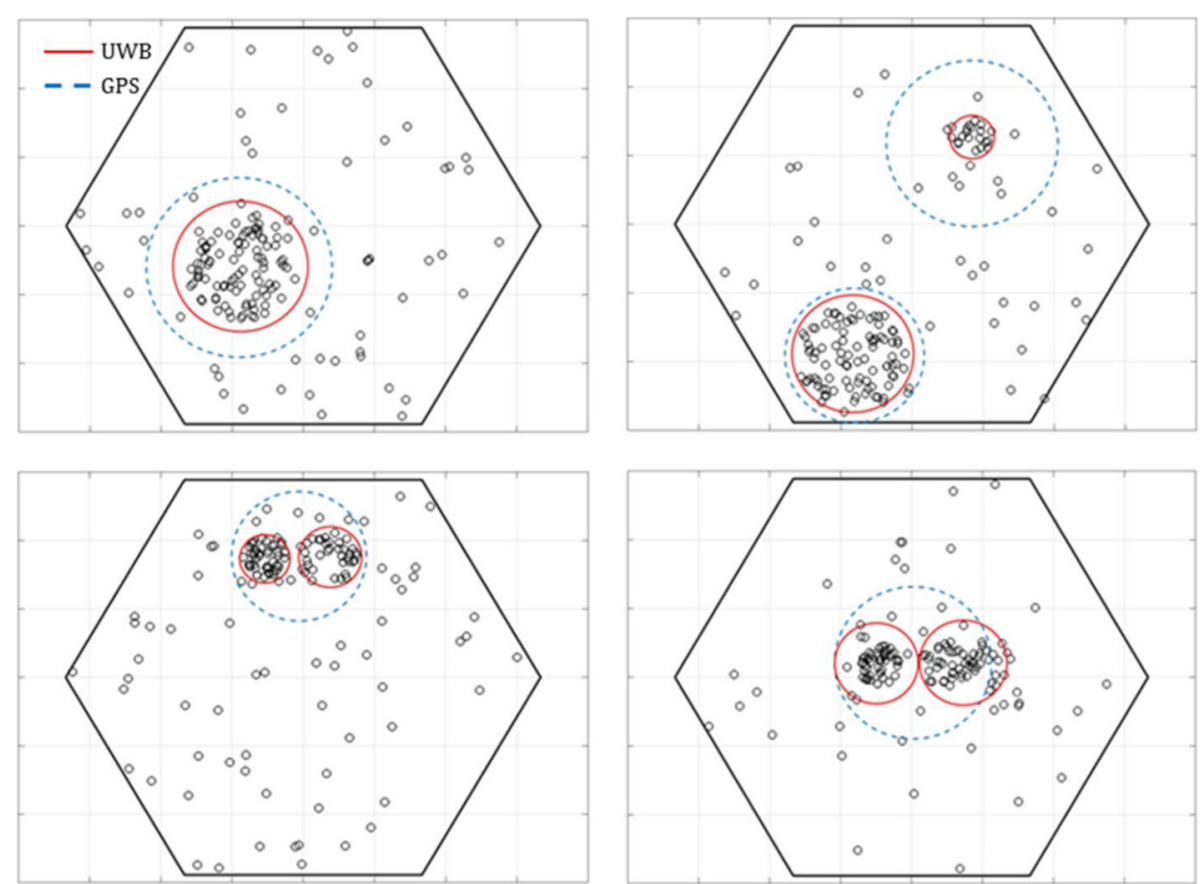

Fig. 15 Four representative clustering realizations. In this figure, four representative clustering realizations depending on UEs distribution are highlighted. Figure 13a (realization i.), GPS and UWB methods give us a hotspot where the one given by UWB signals has a smaller radius than the one given by the GPS. Figure 13b (realization ii.), both clustering methods detect this hotspot, its radius is much smaller using UWB method than GPS and also seems to be more dense. In Fig. 13c (realization iii.) and in Fig. 13d (realization iv.), by using GPS for hotspot detection only the predefined hotspot is detected by the method whereas UWB detects the two sub-hotspots. In Fig. 13d, UEs close to the predefined hotspot are taken into account by the UWB method and are included into the final detection 
dense. Figure $15 \mathrm{c}$,d illustrate a quite similar repartition as in both cases the $60 \%$ of the UEs forming the predefined hotspot of $40 \mathrm{~m}$ radius can actually be separated into two sub-hotspots. By using GPS for hotspot detection, within these two cases, only the predefined hotspot is detected by the method whereas UWB detects the two sub-hotspots. In realization $i v$, UEs close to the predefined hotspot are taken into account by the UWB method, and are included into the final detection. Figure 16 represents the cumulative distribution function (CDF) of the UE density per square meter for the detected hotspots with both methods and we observe that UWB provide much more dense hotspots than GPS method. Also, it is important to note that the hotspots radius are in general smaller using UWB than using GPS as it can be seen in Fig. 17, where the CDF of the hotspots radius is represented for both methods. Generalizing, as more precise the localization system is, the better the clustering results would be. Nevertheless, when selecting localization method, other criteria should also be taken into account such as the complexity, battery consumption, terminal cost, etc. As our objective is to implement VSCs based on hotspot localization, it is important to focus the beamforming signal on a dense and accurate hotspot such that the energy and the resources used while performing the VSCs are optimal.

\section{Conclusions}

This work presents and proposes the concept of VSC as an alternative to classical heterogeneous networks (HetNets) deployed in 4G. Nowadays, the deployment of small cells in $4 \mathrm{G}$ implies a non-negligible cost in terms of new equipment deployment, sites acquisition, and maintenance for operators. In future wireless networks, the use of large

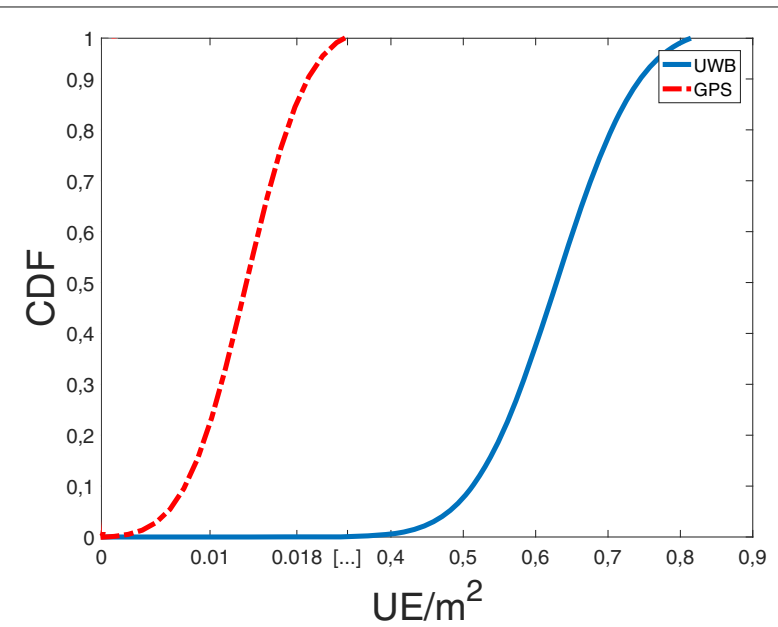

Fig. $16 \mathrm{CDF}$ of the UE density per $m^{2}$ depending on the localization method. This figure represents the CDF of the UE density per square meter for the detected hotspots with both methods and we observe that UWB provide much more dense hotspots than GPS method

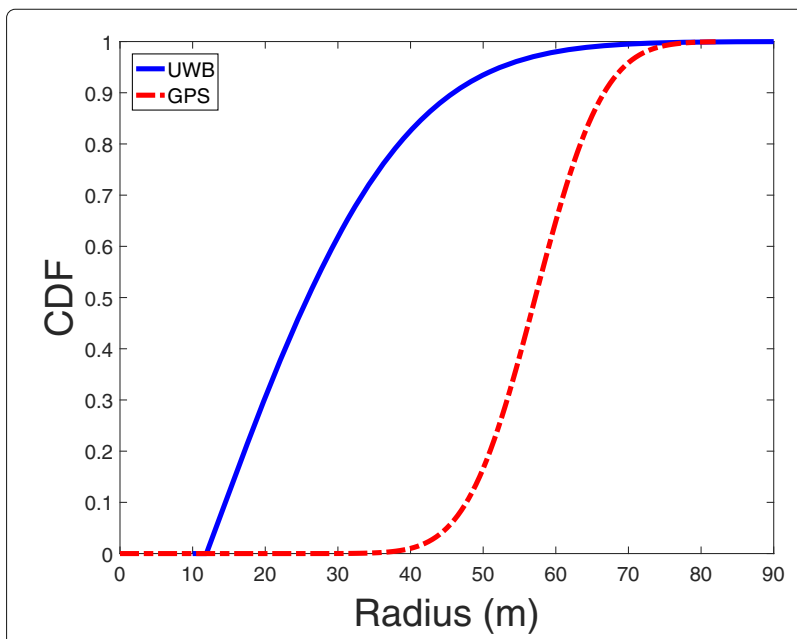

Fig. $17 \mathrm{CDF}$ of the hotspots radius depending on the localization method. This figure represents the CDF of the radius where the hotspots radius are in general smaller using UWB than using GPS

number of antenna elements at the BS will allow the creation of highly directive beams allowing to create VSCs. We plan to use these VSCs in order to replace the typical macro-picocell deployment consequently reducing OPEX and CAPEX expenses. Furthermore, VSCs are reconfigurable during the time and are flexible to changing traffic conditions contrary to typical HetNets deployment. System model and architecture of these VSCs have been mathematically expressed according to informations on hotspots of UEs. System-level simulations show for the case where a VSC is implemented that the average SINR is improved compared to the case where only macrocell is deployed, and achieve most of the time the same performances as classical fix HetNets deployment. Two methods have been proposed in order to dynamically localize the hotspots in the network, one centralized at the BS by using UEs GPS coordinates and a second one independently of the BS, by using D2D communication between the UEs. For the first one, the proposed method shows to correctly detect the optimal number of hotspots at a given time. For the second one, the devices are using UWB signals as it remains the more accurate and less affected by obstacles due to its large band in the high frequency domain. Simulations applying this method show a large improvement of the device localization. Both methods have been compared in an outdoor LOS environment and the UWBbased method provides better accuracy results, error estimation smaller than $1 \mathrm{~cm}$ in $50 \%$ of the cases. The GPS -based localization method can still be used in case where no direct communication between the devices is available. Hotspot localization methods are periodically used in order to dynamically detect new hotspots depending on the traffic and/or adapt their size giving more flexibility in 
terms of coverage and avoiding architecture constraints. Once the hotspots have been successfully localized, a new codebook has been defined based on hotspot information, consequently reducing the feedback to the BS and provides suitable precoding in order to improve the accuracy of the highly directive beams.

\author{
Abbreviations \\ 3GPP: 3rd Generation Partnership Project; BS: Base station; D2D: \\ Device-to-device; GPS: Global Positioning System; LOS: Line of sight; MDT: \\ Minimization of drive tests; MIMO: multiple input multiple output; NLOS: \\ Non-line of sight; ToA: Time of arrival; UE: User equipment; UWB: \\ Ultra-wideband; VSC: Virtual small cell
}

\section{Acknowledgements}

The authors would like to thank Siham Arreffag for her help in the second positioning method.

\section{Funding}

The work is supported by Radio link Innovative DEsign (RIDE) team belonging to Orange Labs Networks, France

\section{Authors' contributions}

TVS made the main contribution on system modeling, codebook design and on methods for positioning. While SML helps out with the second positioning method, AGS supervise the first one. The concept of VSC has been originally propose by both of the co-authors AGS and SML. Finally all authors read and approved the final manuscript.

\section{Competing interests}

The authors declare that they have no competing interests.

\section{Publisher's Note}

Springer Nature remains neutral with regard to jurisdictional claims in published maps and institutional affiliations.

Received: 1 November 2017 Accepted: 4 June 2018

Published online: 26 June 2018

\section{References}

1. H Holma, A Toskala, J Reunanen, LTE small cell optimization: 3GPP Evolution to Release 13. (John Wiley \& Sons, United Kingdom, 2016)

2. J Hoydis, M Kobayashi, M Debbah, Green small-cell networks. IEEE Veh. Technol. Mag. 6(1), 37-43 (2011)

3. A Osseiran, F Boccardi, V Braun, K Kusume, P Marsch, M Maternia, O Queseth, M Schellmann, H Schotten, H Taoka, et al, Scenarios for $5 G$ mobile and wireless communications: the vision of the METIS Pproject. IEEE Commun. Mag. 52(5), 26-35 (2014)

4. S Katikala, Google ${ }^{T M}$ Project Loon. InSight: Rivier Acad. J. 10(2), 1-6 (2014)

5. F Rusek, D Persson, BK Lau, EG Larsson, TL Marzetta, O Edfors, F Tufvesson, Scaling up MIMO: opportunities and challenges with very large arrays. IEEE Signal Proc. Mag. 30(1), 40-60 (2013)

6. S Shalmashi, E Björnson, M Kountouris, KW Sung, M Debbah, Energy efficiency and sum rate tradeoffs for massive MIMO systems with underlaid device-to-device communications. EURASIP J. Wirel. Commun. Netw. 2016(1), 175 (2016)

7. C Shepard, H Yu, N Anand, E Li, T Marzetta, R Yang, L Zhong, in Proceedings of the 18th Annual International Conference on Mobile Computing and Networking. Argos: practical many-antenna base stations (ACM, Istanbul, 2012), pp. 53-64

8. A Adhikary, J Nam, J-Y Ahn, G Caire, Joint spatial division and multiplexing - the large-scale array regime. IEEE Trans. Inf. Theory. 59(10), 6441-6463 (2013)

9. A Galindo-Serrano, SM Lopez, A De Ronzi, A Gati, in Vehicular Technology Conference (VTC Spring), 2015 IEEE 81st. Virtual Small Cells Using Large Antenna Arrays as an Alternative to Classical HetNets (IEEE, Glasgow, 2015), pp. 1-6

10. Y Liu, X Duan, G Boudreau, A Bin Sediq, X Wang, in IEEE Global Communications Conference (GLOBECOM). Adaptive beamforming based inband fronthaul for cost-effective virtual small cell in 5G networks (IEEE, Singapore, 2017)

11. VG Vassilakis, H Mouratidis, E Panaousis, ID Moscholios, MD Logothetis, in 24th IEEE International Conference on Telecommunications (ICT). Security requirements modelling for virtualized $5 \mathrm{G}$ small cell networks (IEEE, Limassol, 2017), pp. 1-5

12. A Radwan, MF Domingues, J Rodriguez, in IEEE International Conference on Communications Workshops (ICC Workshops). Mobile caching-enabled small-cells for delay-tolerant e-Health apps (IEEE, Paris, 2017), pp. 103-108

13. Q Zhang, J Zeng, X Su, L Rong, XXu, in International Conference on Communicatins and Networking in China. Virtual small cell selection schemes based on sum rate analysis in ultra-dense network (Springer, Chongqing, 2016), pp. 78-87

14. T Varela Santana, A Galindo-Serrano, B Sayrac, S Martínez López, in IEEE International Symposium on Wireless Communication Systems (ISWCS). Dynamic network configuration: hotspot identification for virtual small cells (IEEE, Poznan, 2016), pp. 49-53

15. A Dammann, R Raulefs, S Zhang, in IEEE International Conference on Communication Workshop (ICCW). On prospects of positioning in $5 \mathrm{G}$ (IEEE, Shah Alam, 2015), pp. 1207-1213

16. TVarela Santana, S Arreffag, S Martínez López, in IEEE International Symposium on Personal, Indoor and Mobile Radio Communications (PIMRC). A high resolution method for equipment group mapping using UWB signals (IEEE, Montreal, 2017)

17. H Li, L Han, R Duan, GM Garner, Analysis of the synchronization requirements of $5 \mathrm{G}$ and corresponding solutions. IEEE Commun Stand. $\mathbf{1}(1), 52-58(2017)$

18. M Dai, B Clerckx, D Gesbert, G Caire, A rate splitting strategy for massive MIMO with imperfect CSIT. IEEE Trans. Wirel. Commun. 15(7), 4611-4624 (2016)

19. X Rao, VK Lau, Distributed compressive CSIT estimation and feedback for FDD multi-user massive MIMO systems. IEEE Trans. Signal Process. 62(12), 3261-3271 (2014)

20. R Di Taranto, S Muppirisetty, R Raulefs, D Slock, T Svensson, H Wymeersch, Location-aware communications for $5 \mathrm{G}$ networks: how location information can improve scalability, latency, and robustness of $5 \mathrm{G}$. IEEE Signal Proc. Mag. 31(6), 102-112 (2014)

21. S Fortes, A Aguilar-García, R Barco, FB Barba, JA Fernández-Luque, A Fernández-Durán, Management architecture for location-aware self-organizing LTE/LTE-a small cell networks. IEEE Commun. Mag. 53(1), 294-302 (2015)

22. 3GPP TR 36.814 Evolved Universal Terrestrial Radio Access: further advancements for E-UTRA physical layer aspects (release 9). Technical report, 3GPP organization (2010)

23. D Maaz, A Galindo-Serrano, SE EL Ayoubi, in IEEE International Conference on Telecommunication (ICT) Workshop on Main Trends on $5 \mathrm{G}$ and Beyond Networks (MT5Gnet). URLLC User Plane Latency Performance in New Radio (IEEE, Saint Malo, 2018)

24. A Gati, S Martinez-Lopez, T En-Najjary, in Wireless Communications and Networking Conference Workshops (WCNCW). Impact of traffic growth on energy consumption of LTE networks between 2010 and 2020 (IEEE, Istanbul, 2014), pp. 150-154

25. Testing 5G: Data throughput. https://literature.cdn.keysight.com/litweb/ pdf/5992-2519EN.pdf?id=2920715

26. H Yin, L Cottatellucci, D Gesbert, in IEEE 48th Asilomar Conference on Signals, Systems and Computers. Enabling massive MIMO systems in the FDD mode thanks to D2D communications (IEEE, Pacific Grove, pp. $656-660$

27. 3GPP TS 37.320 V11.1.0 Universal Terrestrial Radio Access (UTRA) and Evolved Universal Terrestrial Radio Access (E-UTRA); radio measurement collection for minimization of drive tests (MDT); overall description; stage 2 (Release 11). Technical report, 3GPP organization (2012)

28. J Johansson, WA Hapsari, S Kelley, G Bodog, Minimization of drive tests in 3gpp release 11. IEEE Commun. Mag. 50(11), 36-43 (2012)

29. A Likas, N Vlassis, JJ Verbeek, The global K-means clustering algorithm. Pattern Recognit. 36(2), 451-461 (2003)

30. DT Pham, SS Dimov, CD Nguyen, Selection of K in K-means clustering Proc IME C J Mech Eng Sci. 219(1), 103-119 (2005)

31. 3rd Generation Partnership Project: technical specification group radio access network: study on LTE device to device proximity services; Radio Aspects (Release 12) (2014). 3GPP organization 
32. T Varela Santana, R Combes, M Kobayashi, in IEEE International Symposium on Information Theory (ISIT). Device-to-Device Aided Multicasting IEEE, Vail, Colorado, 2018). June 17-22, 2018

33. R Ibrahim, M Assaad, B Sayrac, A Ephremides, in IEEE International Symposium on Wireless Communication Systems (ISWCS). Overlay D2D vs. cellular communications: a stability region analysis (IEEE, Bologna, 2017)

34. J-W Qiu, CC Lo, C-K Lin, Y-C Tseng, in IEEE Wireless Communications and Networking Conference (WCNC). A D2D relative positioning system on smart devices (IEEE, Istanbul, 2014), pp. 2168-2172

35. N Patwari, JN Ash, S Kyperountas, AO Hero, RL Moses, NS Correal, Locating the nodes: cooperative localization in wireless sensor networks. IEEE Signal Proc. Mag. 22(4), 54-69 (2005)

36. C Savarese, JM Rabaey, J Beutel, in IEEE International Conference on Acoustics, Speech, and Signal Processing, 2001. Proceedings. (ICASSP'01). vol. 4. Location in distributed ad-hoc wireless sensor networks (IEEE, Salt Lake City, UT, 2001), pp. 2037-2040

37. E Karapistoli, F-N Pavlidou, I Gragopoulos, I Tsetsinas, An overview of the IEEE 802.15. 4a standard. IEEE Commun. Mag. 48(1), 47-53 (2010)

38. J Zhang, PV Orlik, Z Sahinoglu, AF Molisch, P Kinney, UWB Systems for wireless sensor networks. Proc. IEEE. 97(2), 313-331 (2009)

39. K Witrisal, S Hinteregger, J Kulmer, E Leitinger, P Meissner, in IEEE International Conference on RFID. High-accuracy positioning for indoor applications: RFID, UWB, 5G, and beyond (IEEE, Orlando, Florida, 2016), pp. 1-7

40. WC Chung, D Ha, in IEEE Conference on Ultra Wideband Systems and Technologies. An accurate ultra wideband (UWB) ranging for precision asset location (IEEE, Reston, Virginia, 2003), pp. 389-393

41. S Gezici, Z Tian, GB Giannakis, H Kobayashi, AF Molisch, HV Poor, Z Sahinoglu, Localization via ultra-wideband radios: a look at positioning aspects for future sensor networks. IEEE Signal Proc. Mag. 22(4), 70-84 (2005)

42. S Al-Jazzar, J Caffery, in IEEE 56th Vehicular Technology Conference. Proceedings. VTC 2002-Fall, vol. 2. ML and Bayesian TOA location estimators for NLOS environments, (2002), pp. 1178-1181

43. S Al-Jazzar, J Caffery, H-R You, A scattering model based approach to NLOS mitigation in TOA location systems, (2002), pp. 861-865

44. B Denis, J Keignart, N Daniele, in Ultra Wideband Systems and Technologies, 2003 IEEE Conference On. Impact of NLOS propagation upon ranging precision in UWB systems (IEEE, Reston, Virginia, 2003), pp. 379-383

45. A Behnad, $X$ Wang, Virtual small cells formation in $5 \mathrm{G}$ networks. IEEE Commun. Lett. 21(3), 616-619 (2017)

46. AF Molisch, K Balakrishnan, C-C Chong, S Emami, A Fort, J Karedal, J Kunisch, H Schantz, U Schuster, K Siwiak, IEEE 802.15. 4a Channel Model-Final Report. IEEE P802. 15(04), 0662 (2004)

47. AF Molisch, P Orlik, Z Sahinoglu, J Zhang, in First International Conference on Communications and Networking in China. ChinaCom'06. UWB-based sensor networks and the IEEE 802.15. 4a Standard-a tutorial (IEEE, Beijing, 2006), pp. 1-6

48. J Zhang, RA Kennedy, TD Abhayapala, Cramér-Rao lower bounds for the synchronization of UWB signals. EURASIP J. Appl. Signal Process. 2005 426-438 (2005)

49. A Shahmansoori, GE Garcia, G Destino, G Seco-Granados, H Wymeersch, Position and orientation estimation through millimeter wave MIMO in $5 \mathrm{G}$ systems. IEEE Trans. Wirel. Commun. 17, 1822-1835 (2017)

50. H Wymeersch, J Lien, MZ Win, Cooperative localization in wireless networks. Proc. IEEE. 97(2), 427-450 (2009)

51. PA Zandbergen, Accuracy of iPhone locations: a comparison of assisted GPS, WiFi and Cellular Positioning. Trans. GIS. 13(s1), 5-25 (2009)

52. G Fischer, O Klymenko, D Martynenko, H Luediger, in International Conference on Indoor Positioning and Indoor Navigation (IPIN). An Impulse Radio UWB Transceiver with High-Precision TOA Measurement Unit (IEEE, Zurich, 2010), pp. 1-8

53. R Merz, F Chastellain, A Blatter, C Botteron, P-A Farine, in European Navigation Conference, Toulouse, France. An experimental platform for an indoor location and tracking system (IEEE, Toulouse, 2008)

54. G Cheng, Accurate TOA-Based UWB Localization system in coal mine based on WSN. Phys. Procedia. 24, 534-540 (2012)

55. M Segura, V Mut, C Sisterna, Ultra wideband indoor navigation system. IET Radar, Sonar \& Navig. 6(5), 402-411 (2012)

\section{Submit your manuscript to a SpringerOpen ${ }^{\circ}$ journal and benefit from:}

- Convenient online submission

Rigorous peer review

- Open access: articles freely available online

- High visibility within the field

- Retaining the copyright to your article

Submit your next manuscript at $\gg$ springeropen.com 